NASA Contractor Report 177980

ICASE REPORT NO. $85-41$

NASA-CR-177980

19860004961

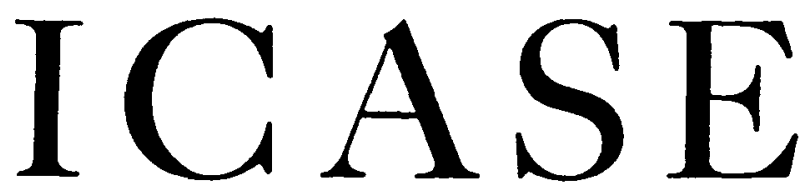

QUALITATIVE PROPERTIES OF LARGE

BUCKLED STATES OF SPHERICAL SHELLS

Ke-Gang Shih

Stuart S. Antman

NASA Contract No. NASI-17070

and 6079

September 1985

Nov 2.5895

MrLlet FEzEARCH CEMTER

LERTRY, MASA

".MOTON, VIRGINIA

INSTITUTE FOR CONPUTER APPLICATIONS IN SCIENCE AND ENGINEERING

NASA Langley Research Center, Hampton, Virginia 23665

Operated by the Universities Space Research Association

\section{N/SA}

National Aeronautıcs and

Space Administratıon

Langley Research Center

Hampton Virginia 23665

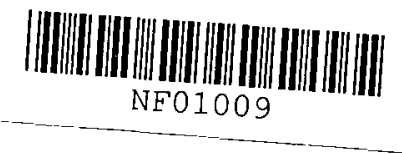




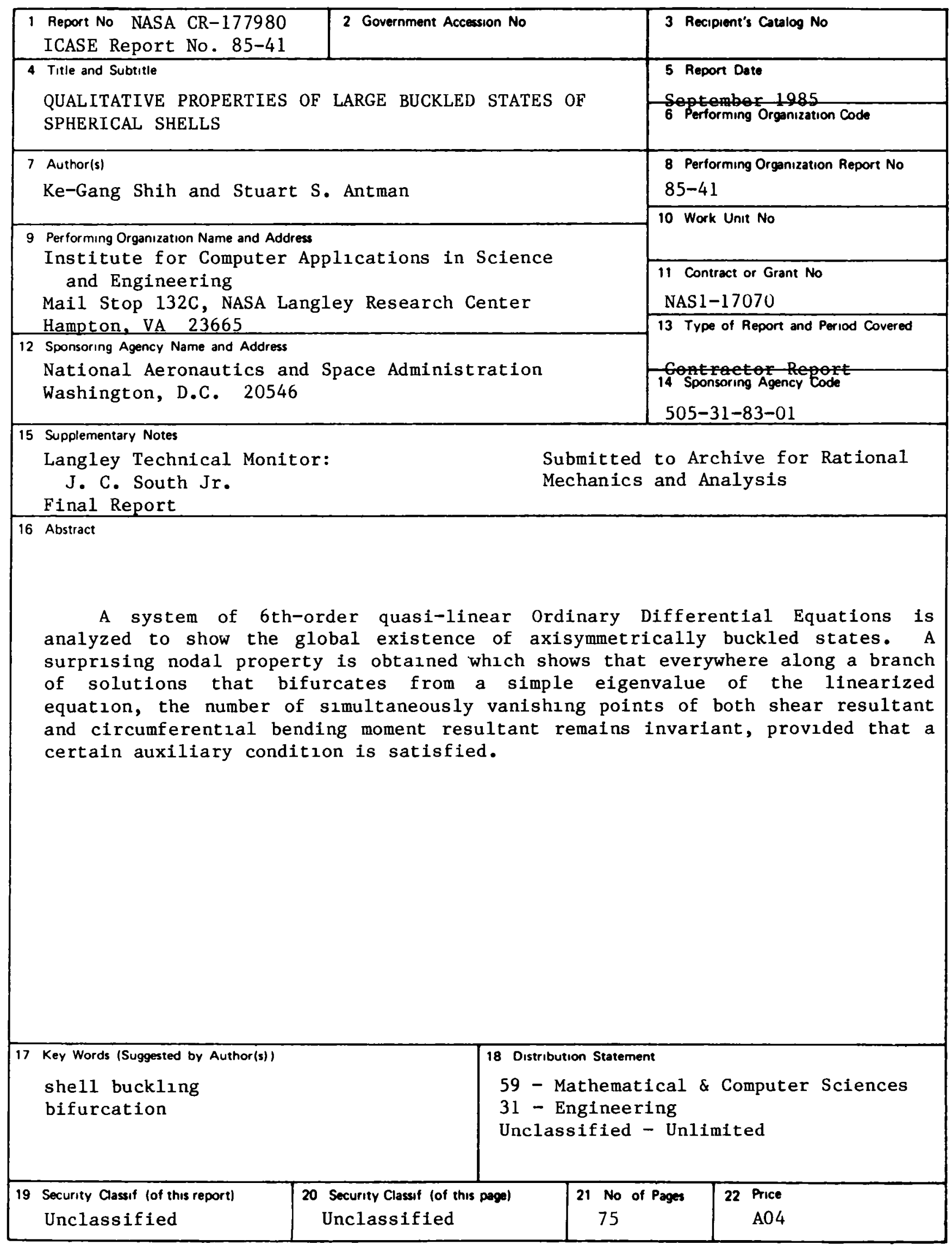




\title{
QUALITATIVE PROPERTIES OF LARGE BUCKLED STATES \\ OF SPHERICAL SHELLS
}

\author{
Ke-Gang Shih \\ Institute for Computer Applications in Science and Engineering
}

Stuart S. Antman

University of Maryland

\begin{abstract}
A system of 6 th-order quasi-linear Ordinary Differential Equations is analyzed to show the global existence of axisymmetrically buckled states. A surprising nodal property is obtained which shows that everywhere along a branch of solutions that bifurcates from a simple elgenvalue of the linearized equation, the number of simultaneously vanishing points of both shear resultant and circumferential bending moment resultant remains invariant, provided that a certain auxiliary condition is satisfied.
\end{abstract}

Research was supported in part by the National Aeronautics and Space Administration under NASA Contract No. NASl-17070 while the author was in residence at ICASE, NASA Langley Research Center, Hampton, VA 23665. 


\section{This Page Intentionally Left Blank}




\section{CONTENTS}

1. Introduction 1-2

2. Formulation of the Governing Equations 3-9

3. Trivial Solutions 10

4. The Linearized Equations 11-18

5. Global Bifurcation 19-31

6. Nudal Properties of Solutions 32-47

7. Properties of $G \quad 48-55$

8. Conclusion 56-58

$\begin{array}{ll}\text { Acknowledgment } & 59\end{array}$

9. Appendix A. Formulation of the Governing 60 Equations

10. References 70 


\section{INTRODUCTINN}

In this paper we study the global qualitatıve behavior of axisymmetric buckled states of homogeneous isotropıc nonlinearly elastic shells that can suffer flexure, compression, and shear. Our model is geometrically exact in the sense that a geometric quantity, such as $\sin \theta$, is not replaced by an approximation, such as $\theta$ or $\theta-\theta^{3} / 6$. (The usual justification for such a replacement is that $\theta$ is known to be small for the physical situation under study. But it is quite possible that a mathematical model with exact geometry permits only small solutions, while those with approximate geometry have large solutions.) we allow the material properties to be described by a very general class of nonlinear constitutive relations. Conseruently our governing equations form a quasilınear sixth-order system of ordınary differential equations.

Our main result is that nontrivial branches of solutions pairs can be globally characterıed by a novel nodal pattern relating shear and bending effects. The detection of this pattern is greatly alded by the generality of our theory, which does not obscure the simple mathematical structure of the governing equations with approximate geometrical relations. Indeed, the beautiful numerical results of Bauer, Reiss, \& Keller (1970) for their technical theory of shells indicate that their solution branches do not enjoy the nodal properties we discover for our exact theory. We surmise that no such technical theory would have these properties. Consequently, 
the nature of the bifurcation diaqram away from the trivial solution for these technical theories could differ markedly from ours.

There is a large literature on the buckling of spherlcal shells. The articles by Bauer, Relss, \& Keller (1970), Antman (1971), Lange \& Kriegsmann (1981), and Shih (1985) give extensive lists of references. Our results differ markedly from those developed in these references (except for the work of Antman) by virtue of the generality of our theory and the global character of our theorems.

The heart of our paper is in Sections 5 and 6 . In Section 5 we show that our equations meet the hypotheses of Rabınowıtz's Global Bifurcation Theorem. In Section 6 we prove a basic uniqueness theorem that enables us to show that solution branches preserve the nodal pattern they inherit from the trivial branch. The use of the isotropy of the shell material is crucial in enabling us to overcome the difficulties posed by the polar singularities.

Notation. Partial derivatives are denoted by subscripts. Ordinary derıvatives with respect to the variable s are denoted by primes. If $f$ and $g$ are functions of $x$ and $y$, then $\frac{\partial(f, q)}{\partial(x, y)}$ denotes the matrix of partial derıvatives of $f$ and $g$ with respect to $x$ and $y$. 
§2. FORMULATION OF THE GOVERNING EQUATIONS.

Let $\{\underset{\sim}{i}, \underset{\sim}{j}, k\}$ be a fixed right-handed orthonormal basis for the Euclidean 3-space $E^{3}$. For each $\phi$ in $R$ we define

$$
\begin{aligned}
& \underset{\sim}{e_{1}}(\phi)=\cos \phi \underset{\sim}{i}+\sin \phi \underset{\sim}{j}, \underset{\sim}{e_{2}}(\phi)=-\sin \phi \underset{\sim}{i}+\cos \phi \underset{\sim}{j}, \\
& \underset{\sim}{e_{3}}=\underset{\sim}{k} .
\end{aligned}
$$

To each $(s, \phi) \in[0, \pi] \times[0,2 \pi]$ there corresponds exactly one point on the sphere of radius 1 centered at the origin with position vector of the form

$$
\underset{\sim}{r}(s, \phi)=\sin s \underset{\sim}{e_{1}}(\phi)-\cos s \underset{\sim}{e_{3}} .
$$

Note that $s$ measures the arc length to $\mathfrak{x}^{*}(s, \phi)$ from the south pole of the sphere. This convention will simplify some of the formulas. We interpret the sphere defined by (2.2) to be the natural reference state of the midsurface of a spherical shell. The coordinates $(s, \phi)$ identify material points on this surface.

An axisymmetric-configuration of a spherical shell that can suffer flexure, extension, and shear is determined by a pair of vector functions

$(2.3) \quad(s, \phi) \mapsto \underset{\sim}{r}(s, \phi), \quad \underset{\sim}{b}(s, \phi)$

with $\underset{\sim}{r}(s, \cdot)$ and $\underset{\sim}{b}(s, \cdot)$ having period $2 \pi$ and with 
$(2.4)$

$$
\begin{aligned}
& \underset{\sim}{r}(s, \phi) \cdot{\underset{\sim}{e}}_{2}(\phi)=0, \\
& \underset{\sim}{b}(s, \phi) \cdot \underset{\sim}{e_{2}}(\phi)=0 . \\
& |\underset{\sim}{b}(s, \phi)|=1 .
\end{aligned}
$$

The reference configuration of the shell is given by $\underset{\sim}{r}=\underset{\sim}{r}{ }_{*} \underset{\sim}{b}=-\underset{\sim}{r} *$. The vector $\underset{\sim}{b}(s, \phi)$ is interpreted as characterizing the deformee configuration of the material points that in the natural state of the shell lie along the ray determined by $\underset{\sim}{r}(s, \phi)$.

We set

$$
\begin{aligned}
\underset{\sim}{r}(s, \phi) & \equiv r(s) \underset{\sim}{e_{1}}(\phi)+z(s) \underset{\sim}{k}, \\
\underset{\sim}{a}(s, \phi) & \equiv \cos \psi(s) \underset{\sim}{e_{1}}(\phi)+s \sin \psi(s) \underset{\sim}{k}, \\
\underset{\sim}{b}(s, \phi) & \equiv-\sin \psi(s) \underset{\sim}{e_{1}}(\phi)+\cos \psi(s) \underset{\sim}{k}, \\
\underset{\sim}{r}(s, \phi) & \equiv v(s) \underset{\sim}{a}(s, \phi)+\eta(s) \underset{\sim}{b}(s, \phi), \\
\tau(s) & \equiv \frac{r(s)}{\sin s}, \\
\mu(s) & \equiv \psi^{\prime}(s)-1, \\
\sigma(s) & \equiv \frac{\sin \psi(s)}{\sin s}-1 .
\end{aligned}
$$

The strain variables for our problem are

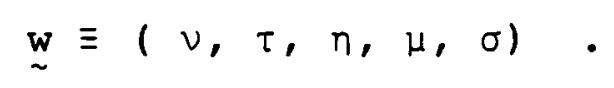

Let ${\underset{\sim}{\mathrm{n}}}_{1}(s, \phi)$ and $\underset{\sim}{\mathrm{m}_{1}}(s, \phi)$ denote the resultant contact force and contact couple per unit reference length 
of the circle $\phi \rightarrow r_{*}(s, \phi)$ of radius $\sin s$ that are exerted across this circular section at $\underset{\sim}{r_{*}}(s, \phi)$. Let $\mathrm{n}_{2}(s, \phi)$ and $\mathrm{m}_{2}(s, \phi)$ denote the resultant contact force and contact couple per unit reference length of the circle $s \rightarrow \underset{\sim}{r}(s, \phi)$ of radius 1 that are exerted across this section at $\underset{\sim}{r}(s, \phi)$. Since we seek axisymmetric solutions, we require these resultants to have the form

$$
\begin{aligned}
& \underset{\sim}{\mathrm{n}_{1}}(s, \phi)=\hat{\mathrm{N}}(s) \underset{\sim}{\mathrm{a}}(s, \phi)+\hat{\mathrm{H}}(s) \underset{\sim}{\mathrm{b}}(s, \phi), \\
& \underset{\sim}{\mathrm{n}_{2}}(s, \phi)=\hat{\mathrm{T}}(s) \underset{\sim}{\underset{2}{e}}(\phi), \\
& \underset{\sim}{\mathrm{m}_{1}}(s, \phi)=-\hat{\mathrm{M}}(s) \underset{\sim}{e_{2}}(\phi), \\
& \underset{\sim}{\mathrm{m}_{2}}(s, \phi)=\hat{\Sigma}(s) \cos \psi(s) \underset{\sim}{e_{1}}(\phi)+\hat{L}(s) \underset{\sim}{k} .
\end{aligned}
$$

Then the equilibrium equations for the shell under an external hydrostatic pressure of intensity $\lambda$ per unit actual area are

(2.14) [sin $\hat{s \hat{N}}(s)]^{\prime}-\hat{T}(s) \cos \psi(s)-\sin s \hat{H}(s) \psi^{\prime}(s)-\lambda r(s) \eta(s)=0$,

(2.15) [sin $\hat{s H}(s)]^{\prime}+\hat{T}(s) \sin \psi(s)+\sin \hat{s \hat{N}}(s) \psi^{\prime}(s)+\lambda r(s) v(s)=0$,

(2.16) [sin $\hat{s M}(s)]^{\prime}-\hat{\Sigma}(s) \cos \psi(s)+\sin s[\nu(s) \hat{H}(s)-n(s) \hat{N}(s)]=0$.

We can combine $(2.14)$ and $(2.15)$ to obtain

(2.17) $\left.\{\sin s \in \sin \psi(s) \hat{N}(s)+\cos \psi(s) \hat{H}(s)]+\lambda r(s)^{2} / 2\right\}^{\prime}=0$, 
(2.18) $\{\sin s[-\cos \psi(s) \hat{N}(s)+\sin \psi(s) \hat{H}(s)]\}^{\prime}+$ $+\hat{T}(s)+\lambda r(s) z^{\prime}(s)=0$.

The material of the shell is homogeneous and nonIinearly elastic if there are constitutive functions $N$, T, H, M, $\Sigma$ such that

$$
\hat{\mathrm{N}}(\mathrm{s})=\mathrm{N}(\underset{\sim}{w}(\mathrm{~s})), \text { etc., }
$$

when $(2.3)-(2.8)$ hold.

We assume that these constitutive functions are thrice continuously differentiable on their common domain of definition

$$
D=\left\{\underset{\sim}{w} \varepsilon R^{5}: \nu>h|\mu+1|, \tau>h|\sigma+1|\right\}
$$

where $h$ is a given number in $(0,1)$, which may be interpreted as half the thickness of the shell. The inequalities in (2.20) ensure that in a standard three-dimensional interpretation of our deformation variables the local ratio of deformed to reference volume never be zero. We require the constitutive functions to satisfy the monotonicity conditions:

$$
\frac{\partial(N, H, M)}{\partial(\nu, \eta, \mu)} \quad, \quad \frac{\partial(T, \Sigma)}{\partial(\tau, \sigma)} \text { are positive-definite. }
$$


( This is the strict form of the strong-ellipticity condition for axisymmetric deformations of axisymmetric shells. Cf. Antman (1978).) These conditions ensure,e.g., that an increase in the bending strain $\mu$ is accompanied by a corresponding increase in the bending couple $M$. We also use special forms of the following very natural inequalities

$$
\mathrm{N}_{\nu}+\mathrm{N}_{\tau}>0, \mathrm{~T}_{\tau}+\mathrm{T}_{\nu}>0 .
$$

We impose compatible growth conditions ensuring that extreme strains are accompanies by extreme resultants:

$$
\begin{aligned}
& \text { (2.23) } \mathrm{N}(\underset{\sim}{w})++\infty \text { as } \nu \rightarrow \infty \text { if } i-h|1+\sigma| \text { has a positive } \\
& \text { lower bound and if } \eta, \mu, \sigma \text { are bounded, } \\
& N(\underset{\sim}{w}) \rightarrow-\infty \text { as } v \rightarrow h|1+\mu| \text { if } \tau \text { is bounded above and } \\
& \text { if } \eta, \mu, \sigma \text {, are bounded, } \\
& T(\underset{\sim}{w}) \rightarrow+\infty \text { as } \tau \rightarrow \infty \text { if } \nu-h|1+\mu| \text { has a positive } \\
& \text { lower bound and if } \eta, \mu, \sigma \text { are bounded, } \\
& T(\underset{\sim}{w}) \rightarrow-\infty \text { as } v \rightarrow h|1+\sigma| \text { if } \nu \text { is bounded above } \\
& \text { and if } \eta, \mu, \sigma \text { are bounded, } \\
& H(\underset{\sim}{w}) \rightarrow \pm \infty \text { as } \eta \rightarrow \pm \infty \text { if }(\nu, \tau, \mu, \sigma) \text { lies in a } \\
& \text { compact subset of }\{(\nu, \tau, \mu, \sigma): v>h|1+\sigma| \text {. } \\
& \tau>h|1+\sigma|\} \\
& M(\underset{\sim}{w}) \rightarrow \pm \infty \text { as } \mu \rightarrow \pm \infty \text { if } \nu>h|1+\sigma| \text { and if }(\tau, n, \mu) \\
& \text { lies in a compact subset of }\{(\tau, \eta, \mu) \text { : } \\
& \tau>h|1+\sigma|\}
\end{aligned}
$$




$$
\begin{aligned}
\sum \underset{\sim}{(w)}+ \pm \infty & \text { as } \sigma \rightarrow \pm \infty \text { if } \tau>h|1+\sigma| \text { and if }(\nu, \eta, \mu) \\
& \text { lies in a compact subset of }\{(\nu, \eta, \mu): \\
& \nu>h|1+\sigma|\} .
\end{aligned}
$$

We finally require that the shell be isotropic and have a stress-free natural state:

$$
\begin{aligned}
& \eta \leftrightarrow H(\underset{\sim}{w}) \text { is odd, } \eta \mapsto N(\underset{\sim}{w}), T(\underset{\sim}{w}), \underset{\sim}{M(w)}, \underset{\sim}{\sum(w)} \text { are even, } \\
& M(\underset{\sim}{w})=0=\sum{\underset{\sim}{w})}_{\sim}^{(w)} \quad \mu=0=\sigma, \\
& N_{\sigma}(\underset{\sim}{w})=N_{\mu}(\underset{\sim}{w})=T_{\sigma}(\underset{\sim}{w})=T_{\mu}(\underset{\sim}{w})=0 \text { if } \eta=\mu=\sigma=0, \\
& N(v, \tau, 0, \mu, \sigma)=T(\tau, v, 0, \sigma, \mu), \\
& M(v, \tau, 0, \mu, \sigma)=\sum(\tau, v, 0, \sigma, \mu), \\
& N(1,1,0,0,0)=T(1,1,0,0,0)=0 .
\end{aligned}
$$

Our boundary conditions essentially require that the deformation be regular at the poles and that there be no net rigid displacement in the $z$-direction :

$(2.25 a, b)$.

$$
r(0)=0=r(\pi) \text {, }
$$

$(2.26 a, b)$

$$
n(0)=0=n(\pi) \text {, }
$$

$(2.27 a, b)$

$$
\psi(0)=0, \psi(\pi)=\pi \text {. }
$$

$$
\int_{0}^{\pi} z(s) d s=0
$$


Substituting (2.25a) into the integral of (2.17) we obtain

(2.29) $\sin s[\hat{N}(s) \sin \psi(s)+\hat{H}(s) \cos \psi(s)]+\lambda r(s)^{2} / 2=0$.

If $\hat{\mathrm{N}}$ and $\hat{\mathrm{H}}$ are bounded and if $\lambda>0$,

then (2.27) implies that $(2.25 b)$ holds. If we further

assume that $\tau$ is bounded, then (2.29) and (2.27) imply

that $\hat{H}(0)=0=\hat{H}(\pi)$. 'The properties of $H$ then ensure

that (2.26) holds. By comparing the integrals of (2.18)

over $[0, s]$ and $[s, \pi]$ we readily obtain

$(2.30)$

$$
\int_{0}^{\pi} \hat{T}(s) d s=-\lambda \int_{0}^{\pi} r(s) z^{\prime}(s) d s .
$$

Note that the integral on the right side is the signed area between the curve $(r, z)$ and the $\underset{\sim}{k}$-axis.

Our boundary value problem consists of the strain configuration equations $(2.5)-(2.8)$, the equilibrium equations (2.14)-(2.16) the constitutive equations (2.19) and the boundary conditions (2.25)-(2.28). A reqular solution of the remaining equations would automatically satisfy $(2.25 b)$ and $(2.26)$.

The shell theory we employ was originated by the Cosserats (1909). (Cf. Naghdi (1972) and Llbal \& Simmonds (1983).) Our formulation was foreshadowed by several papers of Reissner, typical of which is $\mathrm{h}$ is paper of 1963. The variables we employ, and the very simple form of constitutive equations they induce, were introduced by Antman (1971). 
§3. TRIVIAL SOLUTIONS.

We now seek solutions of our boundary value problem in which the shell remains spherical, unsheared, and uniformly stretched, so that $\nu=\tau=k$ (const), $\eta=0, \psi(s)=s$. Thus $\mu=0=\sigma$. Under these conditions, (2.22) reduces $(2.14)-(2.17)$ to

$$
\mathrm{N}(\mathrm{k}, \mathrm{k}, 0,0,0)=\mathrm{T}(\mathrm{k}, \mathrm{k}, 0,0,0)=-\lambda \mathrm{k}^{2} / 2 .
$$

Then (2.22) and (2.23) imply that $k+N(k, k, 0,0,0)+$ $+\lambda \mathrm{k}^{2} / 2$ strictly increases from $-\infty$ to $\infty$ as $\mathrm{k}$ increases from 0 to $\infty$ provided that $\lambda \geqq 0$. Thus (3.1) has a unique solution for $k$, denoted $k(\lambda)$ with

$$
[0, \infty) \geqslant \lambda \rightarrow k(\lambda) \quad \varepsilon(0,1]
$$

thrice continuously differentiable and strictly decreasing . 
§ 4. THE LINEARIZED EQUATIONS.

We adopt the convention that if $R$ is any constitutive function (such as $\left.N, N_{v}, \cdots \cdots\right)$, then

$$
R^{0}(\lambda) \equiv R(k(\lambda), k(\lambda), 0,0,0)
$$

The linearization of our boundary value problem about the trivial solution is equivalent to the following boundary value problem for the linearized variables $v_{1}, n_{1}, \psi_{1}$ :

(4.2) $\begin{aligned} r_{1}(s) & =\int_{0}^{s}\left\{\nu_{1}(t) \cos t-\left[\eta_{1}(t)+k \psi_{1}(t)\right] \sin t\right\} d t= \\ & =\tau_{1}(s) \sin s\end{aligned}$ $=\tau_{1}(s) \sin s$,

(4.3) $\quad N_{\nu}^{0}\left[\left(\nu_{1} \sin s\right)^{\prime}-\tau_{1} \cos s\right]=\left[\left(N_{\tau}^{0}+H_{\eta}^{0}+\lambda k\right) n_{1}+\right.$ $\left.+\left(\mathrm{N}_{\tau}^{0} \mathrm{k}+\lambda \mathrm{k}^{2} / 2\right) \psi_{1}\right] \sin \mathrm{s}$.

(4.4) $\mathrm{H}_{\eta}^{0}\left(n_{1} \sin s\right)^{\prime}=-\left(\mathrm{N}_{\nu}^{0}+\mathrm{N}_{\tau}^{0}+\lambda \mathrm{k}\right)\left(\nu_{1}+\tau_{1}\right) \sin s+$ $+\left(\lambda k^{2} / 2\right)\left(\psi_{1} \sin s\right)^{\prime}$,

(4.5) $M_{\mu}^{0}\left[\left(\psi_{1}^{\prime} \sin s\right)^{\prime}-\psi_{1} / \sin s\right]+\left(M_{\mu}^{0}-M_{\sigma}^{0}\right) \psi_{1} \sin s=$ $=-\left[\mathrm{H}_{\eta}^{0} \mathrm{k}+\lambda \mathrm{k}^{2} / 2\right] \eta_{1} \sin \mathrm{s}$,

(4.6) $\quad n_{1}(0)=0=n_{1}(\pi), \quad \psi_{1}(0)=0=\psi_{1}(\pi)$,

(4.7) $\int_{0}^{\pi} z_{1}(s) d s \equiv \int_{0}^{\pi}\left\{\nu_{1}(t) \sin t+\left[n_{1}(t)+k \psi_{1}(t)\right] \cos t\right\} d t=0$. 
In deriving this system, we relied crucially on (2.24). From this system, we wish to extract a system for $\eta_{1}$ and $\psi_{1}$ alone. To accomplish this efficiently we write (4.3), (4.4), and the derivative of (4.4) in the form $(4.8)$

$$
\begin{aligned}
& \left(\nu_{1} \sin s\right)^{\prime}-\tau_{1} \cos s=f,\left(\nu_{1}+\tau_{1}\right) \sin s=g, \\
& \left(\nu_{1} \sin s\right)^{\prime}+\nu_{1} \cos s=h,
\end{aligned}
$$

from which we immediately deduce that

$$
g=(h-f) \tan s .
$$

We write out this equation, replacing $\left(\psi_{1} \text { 'sin } s\right)^{\prime}-\psi_{1} / \sin s$ by it expression from (4.5) and supplement the resulting equation with $(4.5),(4.6)$ to get the system

$$
\left(L \eta_{1}\right)(s)+A \eta_{1}(s) \sin s=-a \psi_{1}(s) \sin s,
$$

$$
\left(\mathrm{L} \psi_{1}\right)(\mathrm{s})+\mathrm{B} \psi_{1}(\mathrm{~s}) \sin s=-\mathrm{b} \eta_{1}(\mathrm{~s}) \sin s,
$$

$$
n_{I}(0)=0=n_{1}(\pi), \psi_{1}(0)=0=\psi_{I}(\pi),
$$

where

$$
(L u)(s) \equiv\left[u^{\prime}(s) \sin s\right]^{\prime}-u(s) / \sin s,
$$

$$
\begin{aligned}
A= & \left(\mathrm{H}_{\eta}^{0}\right)^{-1}\left[\frac{1}{2} \lambda \mathrm{k}^{2} \mathrm{~b}+\left(\mathrm{N}_{\nu}^{0}+\mathrm{N}_{\tau}^{0}+\lambda k\right)\left(\mathrm{N}_{\nu}^{0}\right)^{-1}\left(\mathrm{~N}_{\tau}^{0}-\mathrm{N}_{\nu}^{0}+\right.\right. \\
& \left.\left.+\lambda k+\mathrm{H}_{\eta}^{0}\right)\right],
\end{aligned}
$$




$$
\begin{aligned}
a= & \left(H_{\eta}^{0}\right)^{-1}\left[\frac{1}{2} \lambda k^{2} B+\left(N_{\nu}^{0}+N_{\tau}^{0}+\lambda k\right)\left(N_{\nu}^{0}\right)^{-1}\left(k N_{\tau}^{0}-\right.\right. \\
& \left.\left.-k N_{\nu}^{0}+\frac{1}{2} \lambda k^{2}\right)\right], \\
B \equiv & 1-M_{\sigma}^{0} / M_{\mu}^{0}, b \equiv\left[H_{n}^{0} k+\lambda k^{2} / 2\right] / M_{\mu}^{0} .
\end{aligned}
$$

We now convert $(4.10),(4.11)$ to a problem for a single complex-valued function

$$
\phi=\alpha \eta_{1}+\beta \psi_{1}
$$

where $\alpha$ and $\beta$ are complex constants to be adjusted so that $(4.10 a, b)$ can be combined into a single complex equation. Thus we require that

$$
(\alpha A+\beta b) n_{1}+(\alpha a+\beta B) \psi_{1}=C \phi=C\left(\alpha \eta_{1}+\beta \psi_{1}\right)
$$

where $C$ is a complex constant to be determined. It follows from (4.15) that

$$
\alpha A+\beta b=\alpha C, \alpha a+\beta B=\beta C
$$

so that

$$
a \alpha^{2}+(B-A) \alpha B-b B^{2}=\dot{0}
$$


Using $(4.15)$ we reduce $(4.10),(4.11)$ to

$$
(L \phi)(s)+C \phi \sin s=0, \phi(0)=0=\phi(\pi),
$$

which has a nontrivial solution if and only if

$$
c=n(n+1), n=1,2, \ldots \ldots
$$

in which case

$$
\phi(s)=\phi_{n}(s)=\text { const. } P_{n}^{1}(\cos s)
$$

where $P_{n}^{1}$ is the associated Legendre function of the first kind of degree $\mathrm{n}$ and order 1 . When (4.19) holds, system (4.16) has a nontrivial solution if and only if

$$
g(\lambda ; n) \equiv[A-n(n+1)][B-n(n+1)]-a b=0 .
$$

Note that A, B, a, b each depneds on the eigenvalue parameter $\lambda$. For a given integer $n$ there can be none, one, or many real solutions $\lambda$ of $(4.21)$. Noting that $N^{0}(\lambda)=-\lambda k(\lambda)^{2} / 2$, we could readily fashion conditions on the constitutive assumptions to ensure any of these possibllities. (Cf. Antman \& Rosenfeld (1978) for a discussion of this issue.) We limit our attention to positive solutions $\lambda$ of (4.21). Negative solutions correspond to shear instabilities. (Cf. 
Antman \& Carbone (1977).)

For $\lambda>0,(2.21)$ implies that $b>0$. Thus (4.16) has no nontrivial solutions $(\alpha, \beta)$ with $\alpha=0$. We can accordingly adopt the special normalization that $\alpha=1$. In this case, the roots $\beta$ of $(4.17)$ are

$$
B_{ \pm} \equiv \frac{B-A \pm \sqrt{(A-B)^{2}+4 a b}}{2 b}=\frac{B-A \pm[A+B-2 n(n+1)]}{2 b},
$$

the second equality coming from (4.21). Thus $B_{ \pm}$are real when (4.21) holds. In this case, $\beta_{ \pm}$are distinct if and only if

$$
A+B \neq 2 n(n+1)
$$

When (4.21) and (4.23) hold, (4.14) and (4.20) reduce to a system of the form

$$
n_{1}+\beta_{ \pm} \psi_{1}=D_{ \pm} P_{n}^{1}(\cos s)
$$

If we take $D_{ \pm}$real and not both 0 , then the distinctness of $\beta_{ \pm}$ensures that (4.24) can be solved uniquely for $n_{1}$ and $\psi_{1}$. These solutions are proportional to $\mathrm{P}_{n}^{1}(\cos s)$. To find $D_{ \pm}$, i.e., to find the constants of proportionality, we normalize $\psi_{1}$ by setting

$$
\psi_{1}(s)=P_{n}^{1}(\cos s), \eta_{1}(s)=E_{n}^{1}(\cos s) .
$$


Then a comparison of (4.10) and (4.11) with (4.18) implies that

$$
A+a / E=n(n+1)=B+b E \text {. }
$$

whence

$(4.27)$

$$
E=\frac{n(n+1)-B}{b}=\frac{a}{n(n+1)-A} \text {. }
$$

The compatibility of these two expressions for $E$ is ensured by $(4.21)$.

Now we study the special case that

$(4.28)$

$$
A+B=2 n(n+1), \quad n=1,2, \ldots .
$$

Then (4.22) implies that $\beta$ is unique with
$(4.29 a, b)$
$2 b B=B-A, b B^{2}=a$

Consequently (4.20) yields

$(4.30)$

$$
n_{1}+\beta \psi_{1}=D P_{n}^{1}(\cos s)
$$

Note that (4.29b) implies that there are no such solutions if $a<0$. 
First we treat the even more special subcase that

$$
A=B=n(n+1)
$$

Then $B=0, n_{1}=\mathrm{DP}_{\mathrm{n}}^{1}(\cos s)$. Moreover $(4.16)$ implies that $a=0$. Since $b>0$, the alternative theorem implies that $(4.10 \mathrm{~b})$ has no solution unless $D=0$ and hence $\eta_{1}=0$. In this case, $\psi_{1}=$ const $P_{n}^{1}(\cos s)$. Note that if we make the eminently reasonable assumption that B $<2$, then (4.31) cannot hold. Likewise, (4.31) cannot hold if $a \neq 0$.

Now we assume that (4.28) holds, but that

$$
A \neq B \neq n(n+1)
$$

Hence $B \neq 0$. Using $(4.30)$ we corvert $(4.10 \mathrm{~b})$ to

$$
L \psi_{1}+n(n+1) \psi_{1}=-b D P_{n}^{1}(\cos s) \sin s
$$

since (4.28) and (4.29) imply that $B-b B=n(n+1)$.

Since $b \neq 0$, the alternative theorem implies that (4.33) has no solution unless $D=0$, in which case $(4.30)$ yields

$(4.34)$

$$
n_{1}=-\beta \psi_{1}
$$


In summary, the eigenvalues of (4.10), (4.11) are solutions $\lambda$ of $(4.21)$. We limit our attention to positive eigenvalues. If $B<2$, then the corresponding eigenfunctions are

(4.35)

$$
\left(n_{1}, \psi_{1}\right)=([n(n+1)-B] / b, 1) P_{n}^{1}(\cos s)
$$

Now the function $P_{n}^{l}(\cos s)$ has exactly $n+1$ zeros on $[0, \pi]$, including those at 0 and $\pi$, each of which is simple. Thus every nontrivial solution $\left(n_{1}, \psi_{1}\right)$ of $(4.10)$, (4.11) is characterized by the fact that $n_{1}$ and $\psi_{1}$ have exactly the same zeros. 


\section{$\S 5$. GLOBAL BIFURCATION .}

To show that our equations meet the hypotheses of the bifurcation theorem and uniqueness theorem, which form the basis of our analysis, we must transform them into alternative forms that enable us to control the polar singularity manifested by the presence of $\sin s$ as a coefficient of the highest derivatives of our differential equations. We substitute (2.19) into (2.14)-(2.17), carry out the differentiation of the leading terms, use Cramer's rule (justified by $(2.21)$ ) to solve the resulting equations for $v^{\prime} \sin s, \eta^{\prime} \sin s, \mu^{\prime} \sin s$, and finally force this latest version of the system into a form suggested by the linear equations (4.3)-(4.5). Using (4.12) we obtain

$$
\begin{aligned}
(\nu \sin s)^{\prime}= & \tau \cos s+\left(N_{\nu}^{0}\right)^{-1}\left(N_{\tau}^{0}+H_{\tau}^{0}+\lambda k\right) n \sin s+ \\
& +\left(N_{\nu}^{0}\right)^{-1}\left(N_{\tau}^{0} k+\lambda k^{2} / 2\right)(\psi-s) \sin s+n,
\end{aligned}
$$

$$
\begin{aligned}
(\eta \sin s)^{\prime}= & -\left(H_{\eta}^{0}\right)^{-1}\left(N_{\nu}^{0}+N_{\tau}^{0}+\lambda k\right)(\nu+\tau) \sin s+ \\
& +\left(H_{n}^{0}\right)^{-1}\left(\lambda k^{2} / 2\right)[(\psi-s) \sin s]^{\prime}+h,
\end{aligned}
$$

where

$$
\begin{aligned}
n \equiv & \Delta^{-1} D_{1}+(\nu-\tau) \cos s-\left(N_{v}^{0}\right)^{-1}\left(N_{\tau}^{0}+H_{\eta}^{0}+\lambda k\right) \eta \sin s- \\
& -\left(N_{v}^{0}\right)^{-1}\left(N_{\tau}^{0} k+\lambda k^{2} / 2\right)(\psi-s) \sin s,
\end{aligned}
$$


$(5.5)$

$$
\begin{aligned}
h \equiv & \Delta^{-1} D_{2}+n \cos s+\left(H_{n}^{0}\right)^{-1}\left(N_{\nu}^{0}+N_{\tau}^{0}+\lambda k\right)(\nu+\tau) \sin s \\
& -\left(H_{n}^{0}\right)^{-1}\left(\lambda k^{2} / 2\right)[(\psi-s) \sin s]^{\prime},
\end{aligned}
$$

$$
\begin{aligned}
m \equiv & \Delta^{-1} D_{3}+(\psi-s)^{\prime} \cos s-\frac{\psi-s}{\sin s}+B(\psi-s) \sin s \\
& + \text { bnsins, }
\end{aligned}
$$

$(5.7 a)$

$$
\Delta \equiv \operatorname{det} \frac{\partial(N, H, M)}{\partial(\nu, \eta, \mu)},
$$

(5.7b) $D_{J}$ is the determinant obtained from $\Delta$ by replacing its jth column with $\left(\begin{array}{l}\alpha \\ \beta \\ \gamma\end{array}\right)$, $\alpha \equiv-\left(\mathrm{N}_{\tau} \tau^{\prime}+\mathrm{N}_{\sigma^{\prime}} \sigma^{\prime}\right) \sin \mathrm{s}+\mathrm{T} \cos \psi-\mathrm{N} \cos \mathrm{s}+\mathrm{H} \psi^{\prime} \sin \mathrm{s}+\lambda r n$,

$$
B \equiv-\left(\mathrm{H}_{\tau} \tau^{\prime}+\mathrm{H}_{\sigma} \sigma^{\prime}\right) \sin s-T \sin \psi-H \cos s-N \psi^{\prime} \sin s-\lambda r \nu,
$$

$$
\gamma \equiv-\left(M_{\tau} \tau+M_{\sigma^{\prime}} \sigma^{\prime}\right) \sin s+\sum \cos \psi-M \cos s-(\nu H-\eta N) \sin s .
$$

From (2.5)-(2.8) we find that

$$
\begin{aligned}
\operatorname{Lr}= & (v \sin s)^{\prime} \cos \psi+(\nu \sin s)(\cos \psi)^{\prime}- \\
& -(n \sin s)^{\prime} \sin \psi-(n \sin s)(\sin \psi)^{\prime}-\tau,
\end{aligned}
$$

$$
\begin{aligned}
\left(z^{\prime} \sin s\right)^{\prime}= & (\nu \sin s)^{\prime} \sin \psi+(\nu \sin s)(\sin \psi)^{\prime}+ \\
& +(\eta \sin s)^{\prime} \cos \psi+(\eta \sin s)(\cos \psi)^{\prime}
\end{aligned}
$$


We now introduce new variables $\left(u_{1}, u_{2}, u_{3}\right) \equiv \underset{\sim}{u}$ by

$$
u_{I}(s)(\sin s)^{\frac{3}{2}}=(\operatorname{Lr})(s), .
$$

$$
u_{2}(s)(\sin s)^{\frac{1}{2}}=\left(z^{\prime} \sin s\right)^{\prime},
$$

$$
u_{3}(s)(\sin s)^{\frac{3}{2}}=I(\psi-s)(s) \quad .
$$

Let us assume that $\underset{\sim}{u}$ is continuous. Then we can solve $(5.11 a, c),(2.25),(2.27)$ in terms of $u_{1}$ and $u_{3}$. Now from (5.11b) we see that $u_{2}$ must satisfy

$$
\int_{0}^{\pi} u_{2}(s)(\sin s)^{\frac{1}{2}} d s=0
$$

We thus find that

$$
\begin{aligned}
z^{\prime}(s) & =(\sin s)^{-1} \int_{0}^{s} u_{2}(t)(\sin t)^{\frac{1}{2}} d t \\
& =-(\sin s)^{-1} \int_{s}^{\pi} u_{2}(t)(\sin t)^{\frac{3}{2}} d t .
\end{aligned}
$$

This representation shows that $z$ satisfies the boundary conditions $z^{\prime}(0)=0=z^{\prime}(\pi)$, which are consequences of $(2.5)-(2.8),(2.26),(2.27)$. We integrate one of the equations of (5.13) and then use (2.28) to evaluate the constant of integration. We obtain

$$
(5.14 a) \quad r(s)=\int_{0}^{\pi} k(s, t) u_{1}(t)(\sin t)^{\frac{1}{2}} d t \equiv\left(G u_{1}\right)(s)
$$


$(5.14 b) \quad z(s)$

$$
\begin{aligned}
& =\int_{0}^{\pi} \frac{\left[\pi^{-1} y-1+x_{[0, s]}(y)\right]}{\sin y} \int_{0}^{y} u^{2}(t)(\sin t)^{\frac{1}{2}} d t d y \\
& \equiv\left(z_{2}\right)(s),
\end{aligned}
$$

$(5.14 c) \quad \psi(s)-s=\int_{0}^{\pi} K(s, t) u_{3}(t)(\sin t)^{\frac{1}{2}} d t \equiv\left(G u_{3}\right)(s)$

where $X_{[0, s]}$ is the characteristic function for $[0, s]$ and $K$ is Green's function for $I$ subject to Dirichlet data:

$(5.15)$

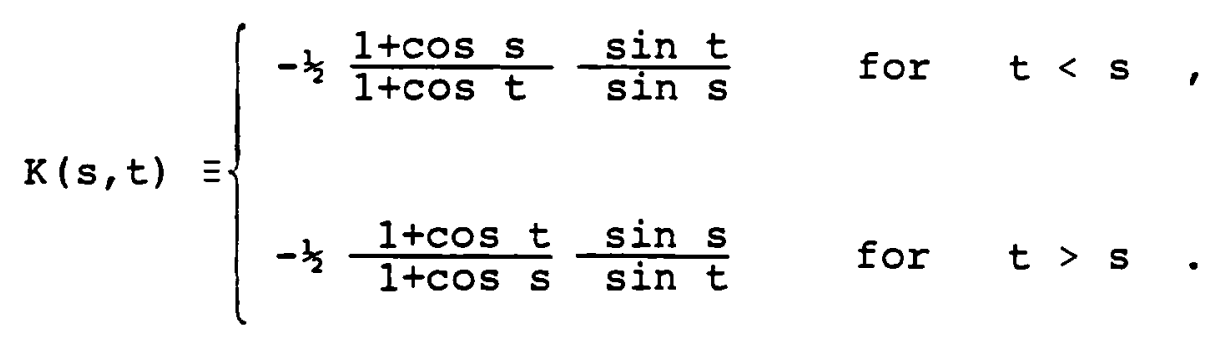

(We could express (5.14b) in terms of a Green's function, but doing so would interfere with our analysis., We also record that

$$
\begin{aligned}
& (5.16 a) \quad I^{\prime}(s)=\int_{0}^{\pi} k_{s}(s, t) u_{1}(t)(\sin t)^{\frac{1}{2}} d t \equiv\left(G u_{1}\right)^{\prime}(s), \\
& (5.16 b) \quad \psi^{\prime}(s)-1=\int_{0}^{\pi} k_{s}(s, t) u_{3}(t)(\sin t)^{\frac{1}{2}} d t \equiv\left(G u_{3}\right)^{\prime}(s)
\end{aligned}
$$

where. 
$(5.17)$

$$
K_{s}(s, t)=\left\{\begin{array}{ll}
\frac{1}{2} \frac{1+\cos s}{1+\cos t} \frac{\sin t}{\sin ^{2} s} & \text { for } t<s, \\
-\frac{1}{2} \frac{1+\cos t}{1+\cos s} \frac{1}{\sin t} & \text { for } t>s
\end{array}\right. \text {. }
$$

$z^{\prime}$ is given by (5.13). From (2.7), (2.8) we then obtain

(5.18a) $\quad v=\left(G u_{1}\right)^{\prime} \cos \left(s+G u_{3}\right)+\left(z u_{2}\right)^{\prime} \sin \left(s+G u_{3}\right)$,

(5.18b) $\quad \eta=-\left(G u_{1}\right)^{\prime} \sin \left(s+G u_{3}\right)+\left(z u_{2}\right)^{\prime} \cos \left(s+G u_{3}\right)$,

$(5.18 \mathrm{c}, \mathrm{d}) \tau=\left(\mathrm{Gu}_{1}\right) / \sin s, \sigma=\left[\sin \left(s+\mathrm{Gu}_{3}\right) / \sin s\right]-1$.

We now convert our differential equations (5.1)(5.3) into integral equations for $\underset{\sim}{u}:$ We replace the left sides of $(5.9),(5.10)$, and $(5.3)$ with $(5.14)$. We next substitute (5.1), (5.2) into the right sides of (5.9), (5.10). Then we replace the variables appearing on the right sides of $(5.9),(5.10),(5.3)$ with their representations from (5.14)-(5.18). Finally, as a precautionary measure, we subtract from the right side of the resulting form of (5.11) its mean value, a move suggested by (5.12). We denote the resulting system of integral equations by

$$
\underline{\sim}=\underset{\sim}{E}(\lambda, \underline{\sim})
$$


Let

(5.20a) $\quad u=\left\{\underline{u} \varepsilon c^{0}([0, \pi])^{3}:(5.12)\right.$ holds. $\}$

We wish to show that $f(\lambda, \cdot)$ is a compact mapping from

$u \cap\{\underset{\sim}{u}: \underset{\sim}{w} \varepsilon D\} \equiv E$

into itself ( $D$ is defined in (2.20)).

Let $\underset{\sim}{w} \rightarrow R(\underset{\sim}{w})$ be any constitutive function. If $R \in C^{1}(D)$, then

$(5.21 a)$

$$
R(\underset{\sim}{y})=R(\underset{\sim}{x})+R_{i}(\underset{\sim}{x} \underset{\sim}{y})\left(y_{i}-x_{i}\right)
$$

with

$(5.21 b)$

$$
R_{i}(\underset{\sim}{x}, \underset{\sim}{y}) \equiv \int_{0}^{1} R_{w_{i}}(t \underset{\sim}{t y}+(1-t) \underset{\sim}{x}) d t .
$$

Here and below, twice repeated indices are summed from 1 to 5. If $R \in C^{2}(D)$, then

$$
R(\underset{\sim}{y})=R(\underset{\sim}{x})+R_{w_{i}} \underset{\sim}{(x)}\left(y_{i}-x_{i}\right)+\frac{1}{2} R_{i j} \underset{\sim}{(x, y)}\left(y_{i}-x_{i}\right)\left(y_{j}-x_{j}\right)
$$

with

$$
R_{i j}(\underset{\sim}{x}, \underset{\sim}{y})=\int_{0}^{1} R_{w_{i} w_{j}}(\underset{\sim}{y}+(1-t) \underset{\sim}{x}) d t .
$$


We let $\bar{R}_{w_{i}} \underset{\sim}{(w)}$ and $\bar{R}_{w_{i} w_{j}}{\underset{\sim}{(w)}}$ be any values of $R_{i}$ and $R_{i j}$ in which the arguments consist solely of entries from $\underset{\sim}{w}$. E.g., $R_{w_{i}}(\underset{\sim}{w})$ could be taken to be $R_{i}((\tau, \nu, 0, \sigma, \mu)$, $(\nu, \tau, n, \mu, \sigma))$

Since we whall use only the continuity of $\left\{\bar{R}_{w_{i}}, \bar{R}_{w_{i} w_{j}}\right\}$ and not the specific functional forms, we allow their meaning to vary even in the same expression.

In the spirit of (5.21) we define the continuously differentiable functions $C$ and $S$ by
$(5.22 a)$

$$
1-\cos \theta=\theta^{2} C(\theta)
$$

$$
\theta-\sin \theta=\theta^{3} s(\theta)
$$

Then
$\cos \psi-\cos s=-(\psi-s) \sin s+U$,
$(5.23 b)$
$U \equiv-(\psi-s)^{2} \mathrm{C}(\psi-s) \cos s+(\psi-s)^{3} s(\psi-s) \sin s$,
$(5,23 c)$
$\sin \psi-\sin s=(\psi-s) \cos s+v$,
$(5.23 d)$

$$
V \equiv-(\psi-s)^{2} C(\psi-s) \sin s-(\psi-s)^{3} s(\psi-s) \cos s
$$

Thus 
$(5.23 e)$

$$
\begin{aligned}
\tau^{\prime} \sin s= & (\nu-\tau) \cos s-(\psi-s)(\nu \sin s+n \cos s)- \\
& -n \sin s+\nu U-n V,
\end{aligned}
$$

$(5.23 f)$

$\sigma^{\prime} \sin s=(\mu-\sigma) \cos s+\psi^{\prime}[U-(\psi-s) \sin s]$.

Let

$$
\underset{\sim}{w^{*}}=(\tau, \nu, 0, \sigma, \mu)
$$

By using the formulas $(5.21)-(5.23)$ we can convert $\alpha$, given by $(5.8 \mathrm{a})$, to

$$
\begin{aligned}
& \alpha=T(\underset{\sim}{w})[U-(\psi-s) \sin s]+\frac{3}{2} \bar{T}_{n \eta} \eta^{2} \cos s- \\
& -\left[N_{\nu}\left(w_{\sim}^{*}\right)(\nu-\tau)+N_{\mu}\left({\underset{\sim}{w}}^{*}\right)(\mu-\sigma)+\frac{1}{2} N_{i j}\left({\underset{\sim}{w}}^{*}, \underset{\sim}{w}\right)\left(w_{i}-w_{i}^{*}\right)\right. \\
& \left.\left(w_{j}-w_{j}^{*}\right)+\bar{N}_{\tau w_{i}}(\nu-\tau)\left(w_{i}-w_{i}^{*}\right)+\bar{N}_{\sigma w_{i}}(\mu-\sigma)\left(w_{i}-w_{1}^{*}\right)\right] \cos s \\
& +\mathrm{N}_{\tau} \underset{\sim}{(w)}[(\psi-s)(v \sin s+n \cos s)+n \sin s-v U+n V]- \\
& -N_{\sigma}(\underset{\sim}{W}) \psi^{\prime}[U-(\psi-s) \sin s]+\bar{H}_{\eta} \eta \psi^{\prime} \sin s+\lambda r \eta \text {. }
\end{aligned}
$$

We obtain similar representations for $B$ and $\gamma$. Note that $\alpha+\left[\mathbb{N}_{\nu}\left({\underset{\sim}{*}}^{*}\right)(\nu-\tau)+N_{\mu}\left(\underset{\sim}{w^{*}}\right)(\mu-\sigma)\right]-\eta\left(\bar{H}_{\eta} \psi^{\prime} \sin s+\lambda r\right)$ consists of sums of product of continuous functions of $(\underset{\sim}{w}, \psi-s)$ with quadratic functions of $\nu-\tau, \eta, \mu-\sigma, \psi-s$. By a lengthy computation we find that the terms in $\underset{\sim}{f}$ consist of products of continuous functions of $(\underset{\sim}{w}, \psi-s)$ with quadratic functions of $(\sin s)^{-\frac{3}{4}}(\nu-\tau),(\sin s)^{-\frac{1}{4}} \eta,(\sin s)^{-\frac{3}{4}}(\mu-\sigma)$, and 
with a number of other such functions of $(\underset{\sim}{w}, \psi-s)$. Let us examine $(\sin s)^{-\frac{1}{4}}(\mu-\sigma)$, which $(5.23 c),(5.14 c),(5.16 b)$ reduce to

$$
\text { (5.26a) } \begin{aligned}
& (\sin s)^{-\frac{1}{4}}\left[\psi^{\prime}(s)-1-(\psi(s)-s) \cot s-V(s) \csc s\right] \\
= & \int_{0}^{\pi} J(s, t) u_{3}(t) d t-(\sin s)^{-5 / 4} V(s)
\end{aligned}
$$

where

$(5.26 b)$

$$
J(s, t)=\left\{\begin{array}{l}
\frac{1}{2} \frac{(1+\cos s)^{2}}{1+\cos t}\left(\frac{\sin s}{\sin t}\right)^{9 / 4}(\sin t)^{-3 / 4}, t<s, \\
-\frac{1}{2} \frac{(1-\cos s)^{2}}{1-\cos t}\left(\frac{\sin t}{\sin s}\right)^{9 / 4}(\sin t)^{-3 / 4}, t>s .
\end{array} .\right.
$$

The Arzela-Ascoli Theorem supports a straightforward proof that the kernel $\mathrm{J}$ generates a compact and continuous operator from $C^{0}([0, \pi])$ to itself. Note that the kernel $(\sin s)^{-\frac{1}{4}} K_{s}($ see $(5.17))$ does not. It is the cancellation of terms in $J$ that is responsible for this compactness. Now (5.23d) implies that

$$
\begin{aligned}
& \text { (5.26c) }-(\sin s)^{-5 / 4} \mathrm{v}=\left[(\sin s)^{-1 / 8}(\psi-s)\right]^{2} c(\psi-s)+ \\
& +\left[(\sin s)^{-5 / 22(\psi-s)}\right]^{3} s(\psi-s) \cos s \text {. }
\end{aligned}
$$

Since the Arzelà-Ascoli Theorem implies that the integral representation for $(\sin s)^{-5 / 12}(\psi-s)$ obtained from $(5.14 \mathrm{c})$ 
in a compact mapping of $\underset{\sim}{u}$, it follows that (5.26c) defines a compact mapping. Since compositions of continuous and compact mappings are compact, we find that (5.26c), $(5.26 a)$, and $(\sin s)^{-1 / 2}(\mu-\sigma)^{2}$ correspond to compact operators taking $C^{0}([0, \pi])$ into itself. We emphasize that the appearance of terms such as $(\mu-\sigma)$ in $f$ is a consequence of the isotropy conditions.

By extensive use of arguments such as these, We find that $f$ is a compact and continuous mapping of $E$ into itself. Moveover, the form of $f$ shows that it is Fréchet differentiable at the trivial branch of solutions. Let $\underline{\sim}_{0}(\lambda)$ be the trivial solution of (5.19) for fixed $\lambda$; $\underline{u}_{0}$ corresponds to the trivial solutions discussed in Section 3. The linearlzation of (5.19) about the trivial state $\underline{u}_{0}$, namely

$$
\underset{\sim}{v}=\underset{\sim}{\underset{v}{v}}\left(\lambda,{\underset{\sim}{u}}_{0}(\lambda)\right) \underset{\sim}{v},
$$

is equivalent to the equations of section 4. We accordingly can invoke the global bifurcation theory of Rabinowitz(1971) and the local theory of Crandall \& Rabinowltz (1971) to obtain

5.28. Theorem. For a given positive integer $n$ let $\bar{\lambda}_{n}$ be a solution of (4.21) of odd algebraic multiplicity,i.e., let there be an odd positive integer $k$ such that 
(5.29) $\quad \frac{\partial^{j}}{\partial \lambda^{j}} g\left(\bar{\lambda}_{n}, \underset{\sim}{u}\right)=0, \quad j=0, \ldots k-1, \quad \frac{\partial^{k}}{\partial \lambda^{k}} g\left(\bar{\lambda}_{n}, \underset{\sim}{u}\right) \neq 0$.

Then bifurcating from the point $\left(\bar{\lambda}_{n}, \sim_{\sim}\left(\bar{\lambda}_{n}\right)\right)$ on the trivial branch of solutions are two connected sets $C^{ \pm}\left(\bar{\lambda}_{n}\right)$ of nontrivial solution pairs $(\lambda, \underset{\sim}{u})$ of (5.19) that enjoy at least one of the following properties :

(i) $\quad c^{ \pm}\left(\bar{\lambda}_{n}\right)$ cannot be confined to any compact subset 요 $E$.

(ii) The closure of $c^{ \pm}\left(\bar{\lambda}_{n}\right)$ contains another point $\left(\zeta, \underline{\sim}_{0}(\zeta)\right)$ where $\zeta$ satisfies (4.21) . Suppose, moreover, that $\bar{\lambda}_{n}$ is simple,i.e., that $k=1$ in (5.29). Let ${\underset{\sim}{\mathrm{V}}}_{\mathrm{n}}$ be a normalized eigenfunction of (5.27). Let $\underline{P}\left(\bar{\lambda}_{n}\right)$ be the projection of $u$ onto span $\left({ }_{\sim n}^{v}\right)$. Then there 2 s a number $\bar{\varepsilon}>0$ and there are continuous functions $\kappa_{n}$ : $[-\bar{\varepsilon}, \bar{\varepsilon}] \mapsto R, \quad{\underset{\sim}{n}}_{n}:[-\bar{\varepsilon}, \bar{\varepsilon}] \mapsto\left[I-P\left(\bar{\lambda}_{n}\right)\right] u$ with $\kappa_{n}(0)=0$, $\underset{\sim}{y}(0)=0$ such that $\left(\bar{\lambda}_{n}+k_{n}(\varepsilon),{\underset{\sim}{u} 0}_{0}\left(\bar{\lambda}_{n}+\kappa_{n}(\varepsilon)\right)+\varepsilon\left[\underline{\sim}_{n}+\underline{\sim}_{n}(\varepsilon)\right]\right)$ is a solution pair of (5.19) for $|\varepsilon|<\bar{\varepsilon}$. Moreover, there is a neighborhood $N_{n}$ of $\left(\bar{\lambda}_{n}, u_{\alpha_{0}}\left(\lambda_{n}\right)\right)$ such that if $(\lambda, \underset{\sim}{u})$ is a solution pair of (5.19) lying in $N_{n}$ ' then either $(\lambda, \underset{\sim}{u})=\left(\lambda, \sim_{0}(\lambda)\right)$ or else there 1s an $\varepsilon \varepsilon[-\bar{\varepsilon}, \bar{\varepsilon}]$ such that $(\lambda, \underset{\sim}{u})=\left(\bar{\lambda}_{n}+k_{n}(\varepsilon),{\underset{\sim}{u}}_{0}\left(\bar{\lambda}_{n}+\kappa_{n}(\varepsilon)\right)+\varepsilon\left[{\underset{\sim}{n}}_{n}+\underset{\sim}{y}(\varepsilon)\right]\right)$.

Note also that (5.14)-(5.18) imply that $\underset{\sim}{w}$ corresponding to any solution of (5.19) is continuous and in 
particular $\psi \varepsilon C^{l}([0, \pi])$. Moreover

$$
\lim _{s \rightarrow 0, \pi}[\nu(s)-\tau(s)]=0, \lim _{s \rightarrow 0, \pi}[\mu(s)-\sigma(s)]=0 .
$$

It follows from (5.1)-(5.3) that $\underset{\sim}{u}$ generates a classical solution to our boundary vlaue problem. In fact, the requirement that the constitutive functions $N, T, H, M, \Sigma$ be thrice continuously differentiable on $D$ implies that the strains $\underset{\sim}{w}$ generated by $\underset{\sim}{u}$ are twice continuously differentiable on the open interval $(0, \pi)$. To determine the boundary behavior of $\eta^{\prime}$, which is needed in the sequel, we divide (2.29) by $\sin ^{2} s$ and use (2.19) and (5.21) to obtain

(5.31) $\quad \mathrm{N}(\underset{\sim}{w}(s)) \frac{\sin \psi(s)}{\sin s}+\bar{H}_{\eta}(\underset{\sim}{w}) \frac{\eta(s)}{\sin s} \cos \psi(s)+\lambda \tau(s)^{2} / 2=0$ on $(0, \pi)$. Since $n$ satisfies $(2.26)$, we can let $s \rightarrow 0, \pi$ in $(5.31)$ to conclude that $n$ is differentiable on $[0, \pi]$. To show that $\eta \in C^{1}([0, \pi])$ we use the expression for $\eta^{\prime}$ obtained from (5.2) and examine its limits as $s \rightarrow 0, \pi$. This process is simplified by the observation that the limiting forms for $\eta^{\prime}$ can equivalently be obtained by substituting (2.19) into (2.15), carrying out the differentiations, dividing the resulting equation by sin $s$, and exploiting $(2.24)$ and (5.30) to show that the limits $\eta^{\prime}(0)$ and $\eta^{\prime}(\pi)$ agree with those found from (5.31). 
An essentially equivalent version of Theorem 5.28 can be obtained by using the theory of Fitzpatrick \& Pejsachowicz (1985), which generalizes that of Crandall and Rabinowitz. The former theory does not require the use of Green functions. The role of isotropystill remains paramount. 
§6. NODAL PROPERTIES OF SOLUTIONS

The determination of nodal properties of solutions of our boundary value problem is a more subtle question than that for the boundary value problem for plates. The main reason for this difference is that in the plate theory (cf. Antman (1978)) $\eta$ is the product of a certain function with $\sin \psi$ and so the nodal properties of $n$ are determined directly from those of $\psi$. On the other hand, in our shell theory $\nu, \tau, \eta, \psi$ are all coupled by nonlinear differential equations, which lack an obvious nodal pattern. To approach the question for shells we first need a candidate for a nodal pattern that is preserved on solution branches.

The solutions of the linearized problem, treated in Section 4 , have the property that $n_{1}$ and $\psi_{1}$ have the same zeros, which are simple. The perturbation solutions of Section 8 also have this feature. Thus we are motivated to study whether the number of simultaneous zeros of $\eta$ and $\psi$ - s is fixed on each bifurcating branch. We immediately confront a serious difficulty. Let $S_{n}, n=1,2, \cdots$ be the collection of all real-valued functions $\rho$ in $C^{1}([0, \pi)]$ having zeros at $0, \pi$ and having exactly $n-1$ zeros on $(0, \pi)$, each zero being simple. Then $S_{n}$ is open in $C^{1}([0, \pi)]$. Given any function in $s_{n}$, it follows that all sufficiently nearby functions enjoy the same nodal properties, i.e., have the 
same number $\mathrm{n}+1$ of zeros, each of which is simple. Moreover, the boundary $\partial S_{n}$ consists of functions having at least one double zero on $[0, \pi]$. These observations support the nodal theory introduced by Crandall \& Rabinowitz (1970) for bifurcation problems governed by nonlinear Sturm-Liouville equations. To study pairs of functions with simultaneous zeros, we let $T_{n}$ be the collection of all complex-valued functions in $\left[C^{1}([0, \pi])\right]^{2}$ having zeros at $0, \pi$ and having exactly $n-1$ zeros on $(0, \pi)$, each zero being simple. But $T_{n}$ is not open in $\left[C^{1}([0, \pi])\right]^{2}$ and there is no useful characterization of $\partial T_{n}$. To circumvent this difficulty, we must exploit the fact that $(n, \psi-s)$ is not merely an arbitrary element of $T_{n}$, but is also a solution of a boundary value problem.

Our strategy is as follows. We first describe an auxiliary condition ensuring that if there is a solution of our boundary value problem for which $\eta^{\prime} \eta^{\prime}, \psi-s$, $(\psi-s)^{\prime}$ all vanish at a point $s_{0}$ in $[0, \pi]$, then $\nu-\tau$ also vanishes at $s_{0}$. Next we prove a basic uniqueness theorem stating that if there is a solution of our boundary value problem to which $\nu-\tau, \eta, \psi-s,(\psi-s)^{\prime}$ all vanish at a point $s_{0}$ in $[0, \pi]$, then the solution is trivial. This result does not enable us to assert that on a nontrivial solution branch $(\eta, \psi-s)$ remains in $T_{n}$ if $(n, \psi-s)$ is in $T_{n}$ at any solution pair on the branch. To remedy this difficulty, we set 
(6.1)

$$
\begin{aligned}
& \eta(s)=\rho(s) \cos \theta(s) \\
& \psi(s)-s=\rho(s) \sin \theta(s) .
\end{aligned}
$$

If $n\left(s_{0}\right)=0=\psi\left(s_{0}\right)-s_{0}$, then $\rho\left(s_{0}\right)=0$. Thus the zeros of the real-valued function $\rho$ are the simultaneous zeros of $\eta$ and $\psi-s$. If $\eta$ and $\psi$ are part of a nontrivial solution of our boundary value problem for which the auxiliary condition holds, we use the uniqueness theorem to prove that $\rho$ can be defined so that it belongs to $C^{1}([0, \pi])$. We can accordingly determine where $\rho \varepsilon \quad S_{n}$. In particular, if at a point on a nontrivial solution branch $\rho \varepsilon S_{n}$, then $\rho$ remains in $S_{n}$ until it has a double zero $($ by virtue of our discussion of $\quad \partial S_{n}$ ).

By the remarks at the beginning of the last paragraph, we conclude that the solution is trivial if $\rho$ has a double zero ( and hence $\eta$ and $\psi$ - s have simultaneous double zeros, provided the auxiliary condition in met. Let $\bar{\lambda}_{n}$ be a simple solution of (4.21) and let $c^{ \pm}\left(\bar{\lambda}_{n}\right)$ be the two nontrivial branches bifurcating from $\left(\bar{\lambda}_{n}, u_{0}\left(\bar{\lambda}_{n}\right)\right)$. We wish to show that on $C^{ \pm}\left(\lambda_{\Omega}\right)$ the function $\rho$ remains in

$S_{n}$ as long as the auxiliary condition is met. To do this, we must show that $\rho$ admits a linearization and enjoys properties like those stated in the last part of Theorem 5.28 . We thereby demonstrate that on $c^{ \pm}\left(\bar{\lambda}_{n}\right)$, the function $\rho$ inherits its nodal properties from those of its linearization and preserves them as long as the auxiliary condition is met, i.e., on $c^{ \pm}\left(\bar{\lambda}_{n}\right), \rho \varepsilon S_{n}$ as long as the auxiliary 
condition is met. Thus on $c^{ \pm}\left(\bar{\lambda}_{n}\right)$ the function $n$ and $\psi-s$ have $n-1$ simultaneous zeros on $(0, \pi)$ as long as the auxiliary condition is met.

We now proceed to carry out this program. We assume throughout the ensuing discussion that $(\lambda, \psi, \underset{\sim}{w})$ generates a solution pair for our boundary value problem.

Suppose that there is an so $\varepsilon(0, \pi)$ such that $\eta\left(s_{0}\right)=\eta^{\prime}\left(s_{0}\right)=\psi\left(s_{0}\right)-s_{0}=\psi^{\prime}\left(s_{0}\right)-1 \equiv \mu\left(s_{0}\right)=0$. Then (2.24) ensures that $(2.15),(2.19),(2.29)$ reduce to

$$
N+T+\lambda \tau\left(s_{0}\right) v\left(s_{0}\right)=0,
$$

$$
N+\frac{1}{2} \lambda \tau\left(s_{0}\right)^{2}=0
$$

where the arguments of $\mathrm{N}$ and $\mathrm{T}$ are $\left(\nu\left(s_{0}\right), \tau\left(s_{0}\right), 0,0,0\right)$. We know from section 3 that $(6.2),(6.3)$ admit a solution of the form $v\left(s_{0}\right)=\tau\left(s_{0}\right)=k(\lambda)$ and that this is the only solution of these equations for which $v\left(s_{0}\right)=\tau\left(s_{0}\right)$. By virtue of $(2.20)$ the variables $\nu, \tau, \lambda$ are confined to $(h, \infty) \times(h, \infty) \times(0, \infty)$. Let $G$ be the subset of this region for which the only solution of $(6.2),(\xi .3)$ is $v\left(s_{0}\right)=\tau\left(s_{0}\right)=k(\lambda)$. We then have the following result, which is virtually a tautology.

6.4. LEMMA. If $\eta\left(s_{0}\right)=\eta^{\prime}\left(s_{0}\right)=\psi\left(s_{0}\right)-s_{0}=\psi^{\prime}\left(s_{0}\right)-1$ $=0$ and if $\left(\nu\left(s_{0}\right), \tau\left(s_{0}\right), \lambda\right) \in G$, then $v\left(s_{0}\right)=\tau\left(s_{0}\right)=k(\lambda)$. 
In the next section we shall study the nature of $G$ the "auxiliary condition", alluded to above is just the requirement that $\left(\nu\left(s_{0}\right), \tau\left(s_{0}\right), \lambda\right) \varepsilon G$. Our basic uniqueness theorem is

6.5. Theorem. If there is an $s_{0} \varepsilon[0, \pi]$ such that

$$
\begin{aligned}
v\left(s_{0}\right)-k(\lambda) & =\tau\left(s_{0}\right)-k(\lambda)=n\left(s_{0}\right)=\psi\left(s_{0}\right)-s_{0}= \\
& =\psi^{\prime}\left(s_{0}\right)-1=0,
\end{aligned}
$$

then the solution is trivial.

Proof. Let

$$
\begin{aligned}
\underset{v}{v} \equiv\left(v_{1}, v_{2}, v_{3}, v_{4}, v_{5}\right) \\
=((v-\tau) \sin s,(v+\tau-2 k) \sin s, n \sin s, \mu \sin s, \psi-s), \\
\quad v=\left|v_{1}\right|+\left|v_{2}\right|+\left|v_{3}\right|+\left|v_{4}\right|+\left|v_{5}\right|
\end{aligned}
$$$$
(6.7 b)
$$

Then we can combine $(5.1)-(5.3),(2.8)$, and $(5.23)$ to obtain the system

$$
v_{1}^{\prime}=v\left(v_{5} \sin s-u\right)+v_{3}+\eta\left(v_{5} \cos s+v\right)+\Delta^{-1} D_{1},
$$$$
v_{2}^{\prime}=\left(v_{1}+v_{2}\right) \cot s-v\left(v_{5} \sin s-u\right)-v_{3}-
$$$$
-\eta\left(v_{5} \cos s+v\right)+\Delta^{-1} D_{1}
$$ 
$(6.8 \mathrm{c})$

$$
v_{3}^{\prime}=v_{3} \cot s+\Delta^{-1} D_{2}
$$

$$
v_{4}^{\prime}=v_{4} \cot s+\Delta^{-1} D_{3},
$$

$$
v_{5}^{\prime}=v_{4}(\sin s)^{-1}
$$

If so $\varepsilon(0, \pi)$ and if $\underset{\sim}{v}$ satisfies $(6.6)$ and $(6.8)$ then $\underset{\sim}{v}=\underset{\sim}{0}$, because the right-hand side of $(6.8)$ is a well-behaved function of $s$ and $\underset{\sim}{v}$. Consequently the initial-value problem has a unique solution, which is the trivial solution $\underset{\sim}{v}=\underset{\sim}{0}$.

Thus we need only concern ourselves with the cases $s_{0}=0, \pi$, in which the right-hand side of $(6.8)$ is singular in $\mathbf{s}$. We just treat the former case. Throughout our discussion we let $\underset{\sim}{v}$ represent an arbitrary, given solution of our boundary value problem ( known to exist by the results of section 5 ) which we subject to the additional requirement that it satisfy $(6.6)$. In the analysis to follow, we let $c$ denote a typical positive constant depending on the fixed but arbitrary solution $\stackrel{v}{v}$. The meaning of $c$ can change from place to place. We tacitly restrict $s$ to lie in an interval of the form $[0, \varepsilon]$ where $\varepsilon$ is small and positive.

since $\sin s \geq s \cos s$ on $[0, \varepsilon]$, we find that

$$
\int_{0}^{s}(\sin t)^{-\frac{1}{2}} d t \leq 2(\sin s)^{\frac{1}{2}} / \cos s \leq c(\sin s)^{\frac{1}{2}} .
$$


Using this result in (5.13)-(5.18) and using the fact that $|\underset{\sim}{u}| \leq c$, we obtain after careful analysis that

$$
\begin{aligned}
& |\nu-\tau|+|n|+|\mu-\sigma| \leqq c(\sin s)^{\frac{1}{2}}, \\
& |\psi-s| \leqq \operatorname{csin} s, \quad|\mu|+|\sigma| \leqq c
\end{aligned}
$$

Since (5.23) implies that

$$
|\sigma| \leqq c\left|v_{5}\right|(\sin s)^{-1}
$$

we find from (5.25) and related expressions that

$$
|\alpha|+|\beta|+|\gamma| \leq c(\sin s)^{\frac{1}{2}}
$$

Let us now examine (6.8a). From (6.10) we immediately obtain

$$
\left|v_{1}^{\prime}-\Delta^{-1} D_{1}\right| \leqq c\left(\left|v_{3}\right|+\left|v_{5}\right|\right) \leqq c v \leqq c v(\sin s)^{-\frac{1}{2}}
$$

Note that (2.21) and (2.23) imply that

$$
\Delta^{-1} \leq c
$$

Accounting for $(2.24)$ by using the notation introduced after (5.21) we obtain 
$(6.15)$

$$
\begin{gathered}
D_{1}=\alpha \mathrm{H}_{\eta} \mathrm{M}_{\mu}-\alpha \overline{\mathrm{H}}_{\mu \eta} \overline{\mathrm{M}}_{\eta \eta} \eta^{2}-\left(\beta \mathrm{M}_{\mu}-\gamma \mathrm{H}_{\mu}\right) \overline{\mathrm{N}}_{\eta \eta} \eta+ \\
+\mathrm{BN}_{\mu} \overline{\mathrm{M}}_{\eta \eta} \eta-\gamma \mathrm{H}_{\eta}\left(\overline{\mathrm{N}}_{\mu \eta} \eta+\overline{\mathrm{N}}_{\mu \mu} \mu+\overline{\mathrm{N}}_{\mu \sigma} \sigma\right),
\end{gathered}
$$

$$
\Delta^{-1}-\left(\mathrm{N}_{\nu} \mathrm{H}_{n} \mathrm{M}_{\mu}\right)^{-1} \equiv \mathrm{Q}=\overline{\mathrm{Q}}_{\eta}{ }^{-1}+\overline{\mathrm{Q}}_{\mu} \mu+\overline{\mathrm{Q}}_{\sigma} \sigma
$$

Inequalities $(6.10)-(6.12),(6.14)-(6.16)$ imply that

$$
\left|\Delta^{-1} D_{1}-\alpha\left(N_{v}\right)^{-1}\right| \leq c v(\sin s)^{-\frac{1}{2}}
$$

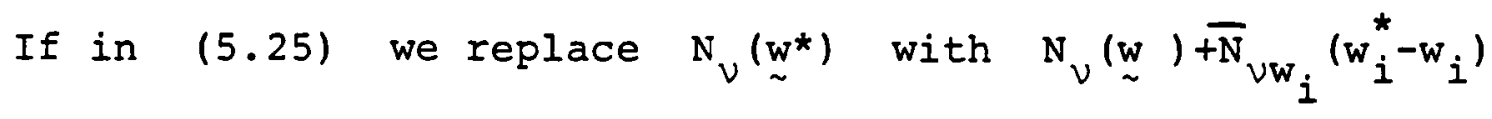
and replace $\mathrm{N}_{\mu}\left(w_{\sim}^{*}\right)$ with $\overline{\mathrm{N}}_{\mu \eta} n^{n+\overline{\mathrm{N}}_{\mu \mu}} \mu+\overline{\mathrm{N}}_{\mu \sigma} \sigma$, then we obtain from $(6.10),(6.11)$ that

$$
\left|\alpha+N_{\nu}(\nu-\tau) \cos s\right| \leq c v(\sin s)^{-\frac{1}{2}}
$$

Inequalities $(6.13),(6.17),(6.18)$ imply that

$$
\left|v_{1}^{\prime}+v_{1} \cot s\right| \leqq c v(\sin s)^{-\frac{3}{2}}
$$

A virtually identical analysis yields

$$
\left|v_{2}^{\prime}-v_{2} \cot s\right| \leq c v(\sin s)^{-\frac{1}{2}}
$$

The treatment of $(6.8 \mathrm{c})$ is just slightly different. Using the same ideas as those leading to (6.17) we obtain 
$(6.21)$

$$
\left|\Delta^{-1} D_{2}\right| \leqq C|B|+C v(\sin s)^{-\frac{1}{2}}
$$

Since $\mathrm{H}_{\tau}=\overline{\mathrm{H}}_{\tau \eta} \eta, \mathrm{H}_{\sigma}=\overline{\mathrm{H}}_{\sigma \eta} \eta$ by (2.24), equation (5.8) yields

$$
|B| \leqq c v(\sin s)^{-\frac{1}{2}}+|T+N+\lambda \nu \tau| \sin s \quad .
$$

Equation (3.1) Implies that

$$
\begin{aligned}
T+N+\lambda \tau \nu & \equiv x=\bar{x}_{\nu}(\nu-k)+\bar{x}_{\tau}(\tau-k) \\
& =\frac{1}{2}\left[\bar{x}_{v}\left(v_{1}+v_{2}\right)+\bar{x}_{v}\left(-v_{1}+v_{2}\right)\right](\sin s)^{-1}
\end{aligned}
$$

Combining $(6.8 \mathrm{c}),(6.21)-(6.23)$, we obtain

$$
\left|v_{3}^{\prime}-v_{3} \cot s\right| \leq c v(\sin s)^{-\frac{1}{2}}
$$

We remark that the only place that the variable $v_{2}$ appears in $(6.8 a, c, d, e)$ is in the right side of $(6.8 c)$. Similar techniques produce

$$
\left|v_{4}^{\prime}-v_{5} \cot s\right| \leqq c v(\sin s)^{-\frac{1}{2}}
$$

Combining $(6.19),(6.20),(6.24),(6.25),(6.8 e)$ we obtain

$$
v^{\prime} \leqq\left|v_{1}^{\prime}\right|+\left|v_{2}^{\prime}\right|+\left|v_{3}^{\prime}\right|+\left|v_{4}^{\prime}\right|+\left|v_{5}^{\prime}\right| \leqq v \operatorname{vcot} s+c v(\sin s)^{-\frac{2}{2}},
$$


the first inequality being a standard consequence of the absolute continuity of $\underset{\sim}{v}$. Since (6.6) implies that

$$
v(s) / s \rightarrow 0 \text { as } s \rightarrow 0,
$$

we can invoke the standard uniqueness theorems associated with differential inequalities (cf. Hartman(1964, Sec.III.6)) to conclude that $v=0$. 0

It is important to note that if we were to insert a constant exceeding 1 in front of vcot $s$ in (6.26), then we could not conclude that $v=0$. That the coefficient in front of vcot $\mathbf{s}$ is 1 is a consequence of the isotropy condition. 
We are now ready to study the polar coordinates $\rho$ and $\theta$ introduced in (6.1). We state their properties in a series of lemmas.

6.28. Lemma. Let the boundary value problem have a nontrivial solution pair with $(\nu, \tau, \lambda)$ taking values only in $G$ and with $P$ and $\theta$ defined in any manner consistent with (6.1). Then the zeros of $\rho$ on $[0, \pi]$ are isolated. Proof. If not, they would accumulate at a point $s_{0} \varepsilon[0, \pi]$, at which $n$ and $\psi-s$ would consequently have simultaneous double zeros. By Lemma 6.4 and Theorem 6.5, the solution would then be trivial. 0

Now (6.1) implies that

$$
\tan \theta=(\psi-s) / n .
$$

Suppose that the solution is not trivial and that $(\nu, \tau, \lambda)$ take values only in $G$. Let $s_{1}$ and $s_{2}$ be a pair of adjacent zeros of $\rho$. If $n$ vanishes nowhere on $\left(s_{1}, s_{2}\right)$, then (6.29) implies that $\tan \theta$ is continuously differentiable on $\left(s_{1}, s_{2}\right)$ and we can define $\theta$ itself (mod $\pi$ ) to be continuously differentiable on $\left(s_{1}, s_{2}\right)$. If $\eta$ vanishes at $s_{3} \varepsilon\left(s_{1}, s_{2}\right)$, then we define 
(6.30) $\theta(s) \equiv \pi / 2+\int_{s_{3}}^{s} \frac{\left\{n(t)\left[\psi^{\prime}(t)-1\right]-[\psi(t)-1] \eta^{\prime}(t)\right\}}{n(t)^{2}+[\psi(t)-t]^{2}} d t(\bmod \pi)$

for $s \in\left(s_{1}, s_{2}\right)$. If $s_{4}$ is a zero of $\rho$ and if $\eta^{\prime}\left(s_{4}\right)$ and $\psi^{\prime}\left(s_{4}\right)-1$ do not both vanish, then I'Hopital's rule enables us to deduce from (6.29) that

$$
\tan \theta(s)+\frac{\psi^{\prime}\left(s_{4}\right)-1}{\eta^{\prime}\left(s_{4}\right)} \text { as } s \rightarrow s_{4} \text {, }
$$

the limit existing as an extended real number. (If $n^{\prime}\left(s_{4}\right)=$ $=0=\psi^{\prime}\left(s_{4}\right)-1$, then we could use Lemma 6.4 and Theorem 6.5 to show that the solution is trivial when the condition on $G$ holds.) Then $\theta\left(s_{4}\right)$ is defined mod $\pi$. By selectina the value of $\theta$ at such zeros of $\rho$ appropriately, we can define

$$
\rho=n \cos \theta+(\psi-s) \sin \theta .
$$

Hence Lemma 6.4 and Theorem 6.5 imply

6.33. Lemma. Let the boundary value problem have a nontrivial solution pair with $(\nu, \eta, \lambda)$ taking values only in $G$ Then $\rho$ and $\theta$ satisfying $(6.1)$ can be chosen to be continuous on $[0, \pi]$.

Since $\eta, \psi \in C^{1}([0, \pi])$, the constructions we have made above ensure that $\rho$ and $\theta$ are continuously differen- 
tiable where $\rho$ does not vanish provided the hypotheses of Lemma 6.33 hold. Differentiating (6.1) we obtain

$$
\begin{aligned}
(6.34 a, b) \quad \rho^{\prime} & =\eta^{\prime} \cos \theta+\left(\psi^{\prime}-1\right) \sin \theta, \\
\rho \theta^{\prime} & =-\eta^{\prime} \sin \theta+\left(\psi^{\prime}-1\right) \cos \theta
\end{aligned}
$$

where $\rho$ does not vanish. The continuity of the right sides of $(6.34)$ then yields

6.35. Lemma. If the hypotheses of Lemma 6.33 hold, then $\rho^{\prime}, \rho \theta^{0} \varepsilon C^{0}([0, \pi])$.

We require the following refinement of this lemma :

6.36. Lemma. If $s_{0}$ is a zero of $\rho$ and if $\eta^{\prime}\left(s_{0}\right)$ and $\psi^{\prime}\left(s_{0}\right)^{-1}$ are not both zero, then $\rho\left(s_{0}\right) \theta^{\prime}\left(s_{0}\right)=0$.

Proof. The Mean Value Theorem yields

$(6.37)$

$$
\begin{aligned}
\eta(s) & =\left(\int_{0}^{1} \eta^{\prime}\left(\alpha s+(1-\alpha) s_{0}\right) d \alpha\right)\left(s-s_{0}\right), \\
\psi(s)-s & \left.=\iint_{0}^{1} \mu\left(\alpha s+(1-\alpha) s_{0}\right) d \alpha\right)\left(s-s_{0}\right) .
\end{aligned}
$$

On any small one-sided neighborhood of $s_{0},(6.34 \mathrm{~b})$ thus yield 


$$
\begin{aligned}
& (6.38) \quad \pm \rho(s) \theta^{\prime}(s) \\
& =\frac{\mu(s) \int_{0}^{1} \eta^{\prime} d \alpha-\eta^{\prime}(s) \int_{0}^{1} \mu d \alpha}{\left(\left[\int_{0}^{1} \eta^{\prime} d \alpha\right]^{2}+\left[\int_{0}^{1} \mu d \alpha\right]^{2}\right)^{\frac{1}{2}}}
\end{aligned}
$$

where the arguments of $\eta^{\prime}$ and $\mu$ in the integrands are $\alpha s+(1-\alpha) s_{0}$. Since $\eta^{\prime}$ and $\mu$ are unlformly continuous on $[0, \pi]$, we find that $\int_{0}^{1} \eta^{\prime}\left(\alpha s+(1-\alpha) s_{0}\right) d \alpha \rightarrow \eta^{\prime}\left(s_{0}\right)$ as $s \rightarrow s_{0}$, etc. Thus in the limit as $s \rightarrow s_{0}$, the numerator on the right side of (6.38) approaches 0 , while the denominator approaches a positive number . 0

In view of Lemma 6.35 we can examine whether $\rho \varepsilon S_{n}$. By the introductory remarks of this section, functions $\rho$ in $\partial S_{n}$ have a double zero. But this fact alone does not guarantee that $n$ and $\psi-s$ have a simultaneous double zeros, as an examination of (6.1) and (6.34a) shows. But Lemma 6.36 coupled with (6.34) implies that at a double zero of $\rho$ the functions $\eta$ and $\psi-s$ have a simultaneous double zero, a result that enables us to invoke Lemma 6.4 and Theorem 6.5 . Marshalling these facts in the manner proposed in the introductory remarks in this section, we obtain

639. Theorem. Let $K$ be a connected set of nontrivial solution pairs with the property that $(\nu, \tau, \lambda)$ takes values 
only in $G$ for each solution pair in $K$. If for one solution pair in $K$ the function $\rho \varepsilon S_{n}$, then $\rho$ remains in $S_{n}$ for every solution pair in $k$. Thus $n$ and $\psi-s$ have exactly $n+1$ simultaneous zeros on $k$.

Let $\bar{\lambda}_{n}$ be a simple root of (4.21) (so that (5.29) holds with $k=1$ ). Then the last part of Theorem 5.28 together with the representations (5.14c) and (5.18b) imply that on the branch $c\left(\bar{\lambda}_{n}\right)$ near the bifurcation point $\left(\bar{\lambda}_{n}, \underset{\sim}{0}\right), \lambda, n, \psi$ have representations of the form

$(6.40)$

$$
\begin{aligned}
& \lambda=\bar{\lambda}_{n}+\kappa(\varepsilon), \eta=\varepsilon \eta_{1}+o(\varepsilon), \\
& \psi-s=\varepsilon \psi_{1}+o(\varepsilon)
\end{aligned}
$$

as $\varepsilon \rightarrow 0$, where $K$ is continuous and $k(0)=0$. If we substitute $(6.40)$ into (6.29) and use (4.25) we obtain

$(6.41)$

$$
\tan \theta=\psi_{1} / \eta_{1}+o(1)=E^{-1}+o(1) .
$$

Thus we may take

$$
\theta=\arctan \left(E^{-1}\right)+o(1) .
$$

Then (6.32) implies that

$$
\rho=\varepsilon \rho_{1}+o(\varepsilon)=\varepsilon\left[\rho_{I}+O(\varepsilon) \varepsilon^{-1}\right]
$$


with

(6. 44$)$

$$
\begin{aligned}
\rho_{1} & =(1+E)^{-\frac{1}{2}}\left[E \eta_{1}+\psi_{1}\right] \\
& =(1+E)^{\frac{1}{2}} P_{n}^{1}(\cos s) .
\end{aligned}
$$

It follow that $\rho_{1} \quad \varepsilon \quad S_{n}$ and that $\rho \varepsilon \quad S_{n}$ for $\varepsilon$ sufficiently small. (Incidentally, we show in the next section that $(\nu, \tau, \lambda)$ generically belongs to $G$ for $\varepsilon$ sufficiently small essentially because $\nu-\tau=0$ on the trivial branch. ) We accordingly have

6.45. Theorem. Let $\bar{\lambda}_{n}$ be a simple root of $(4.21)$. On the maximal connected subset of the closure of $c^{ \pm}\left(\bar{\lambda}_{n}\right)$ containing $\left(\bar{\lambda}_{n}, \underline{\sim}_{0}\left(\bar{\lambda}_{n}\right)\right)$ for which $(\nu, \tau, \lambda)$ takes values only in $G, n$ and $\psi$-s have exactly $n+1$ simultaneous zeros. 
§7. PROPERTIES OF $G$.

We now study $(6.2),(6.3)$, which we rewrite as

$$
N(\nu, \tau)=-\frac{1}{2} \lambda \tau^{2},
$$

$$
N(\nu, \tau)+T(\nu, \tau)=-\lambda \nu \tau,
$$

from which we obtain

$$
T(\nu, \tau)=\lambda \tau\left(\frac{1}{2} \tau-\nu\right)
$$

Here we have suppressed the last three arguments $(0,0,0)$ of the functions $N$ and $T$. The set $G$ consists of all $(\nu, \tau, \lambda)$ in $(h, \infty) \times(h, \infty) \times(0, \infty)$ for which the only solution of $(7.1),(7.2)$ is $\nu=\tau=k(\lambda)$. We make a few remarks about (7.1), (7.2) in general before studying a specific example.

$$
\text { Since } N_{V}>0 \text { by }(2.21) \text { and since } N \text { satisfies }
$$
the growth conditions of (2.23), equation (7.1) can be solved uniquely for $\nu$ in terms of $\tau$ and $\lambda$ :

$$
\nu=f(\tau ; \lambda), \quad \text { with } 1=f(1 ; 0) .
$$

Since $f_{\tau}=-\left(\mathrm{N}_{\tau}+\lambda \tau\right) / \mathrm{N}_{\nu}$, we find that $\mathrm{f}_{\tau}<0$ if $\mathrm{N}_{\tau}>0$ and that $f_{\tau}(1 ; 0)>-1$ if $N_{\tau}(1,1)<N_{v}(1,1)$. The isotropy condition (2.24) ensures that the curve in the $(\nu, \tau)$-plane defined by $(7.2)$ is symmetric 
about the line $v=\tau$. If $\mathrm{T}_{\nu}+\mathrm{N}_{\nu}>0$, if $\mathrm{T}+\mathrm{N} \rightarrow-\infty$ as $v \rightarrow 0$ for fixed $\tau$, and if $\liminf T+N \geqq 0$ as $\nu \rightarrow \infty$ for fixed $\tau$, then $(7.2)$ can be uniquely solved for $\nu$ in terms of $\tau$ and $\lambda$ :

$$
\nu=g(\tau ; \lambda) \text { with } 1=g(1 ; 0) \text {. }
$$

The results of section 3 ensure that the graphs of $f(\cdot ; \lambda)$ and $g(\cdot ; \lambda)$ intersect on the line $\nu=\tau$. Since

$$
g_{\tau}=-\frac{\left(\lambda g+N_{\tau}+T_{\tau}\right)}{\left(\lambda_{\tau}+N_{\nu}+T_{\tau}\right)}
$$

it follows that $g_{\tau}<0$ under our assumptions and, not surprisingly, that $f_{\tau}(\tau ; \lambda)=-1$ when $\tau=g(\tau ; \lambda)$. Thus the graphs of $f(\cdot ; \lambda)$ and $g(\cdot ; \lambda)$ cross transversally on the line $v=\tau$ when $\mathrm{f}_{\tau} \neq-1$ here, i.e., when

$$
\mathrm{N}_{\nu}(\tau, \tau)-\mathrm{N}_{\tau}(\tau, \tau)-\lambda \tau \neq 0
$$

Using (7.1) we can rewrite $(7.7$ a) as a condition involvIng only the constitutive function $\mathrm{N}$ :

$$
N_{v}(\tau, \tau)-N_{\tau}(\tau, \tau)+2 N(\tau, \tau) / \tau \neq 0
$$

Wherever (7.7) holds, there is a neighborhood about $(\tau, \tau, \lambda)$ lying in $G$. ( see the parenthetical remark 
preceding Theorem 6.45.)

There are several ways to analyze (7.1), (7.2) further by making general restrictions on $N$ and $T$ leading to suitable estimates. It is also illuminating to view (7.1) and (7.2) as each describing the intersection of two graphs over the $(\nu, \tau)$-plane. Alternatively we could assume that $\mathrm{N}$ and $\mathrm{T}$ are derivable from $a$ stored energy function ( as in (7.9) below), characterize the equations as Euler-Lagrange equations for a constrained problem, and study the extremization of a real valued function on a constraint curve. There are several ways to choose the constraint. It is more illuminating, however, to analyze a specific class of materials.

Let $A, B, C, \alpha$ be positive numbers and let

$$
W(\nu, \tau)=A \alpha^{-1} \nu-\alpha \tau^{-\alpha}+\frac{1}{2} B(\nu+\tau)^{2}+\frac{1}{2} C\left(\nu^{2}+\tau^{2}\right)
$$

$$
\begin{aligned}
& N(\nu, \tau)=w_{\nu}(\nu, \tau)-w_{\nu}(1,1) \\
& T(\nu, \tau)=w_{\tau}(\nu, \tau)-w_{\tau}(1,1)
\end{aligned}
$$

These equations give a reasonable model for material behavior in compression for $h=0$. When (7.8), (7.9) hold, equations $(7.1),(7.2)$ reduce to

$$
-A \nu^{-(\alpha+1)} \tau-(\alpha+1) \tau+B(\nu+\tau)+C \nu+A-2 B-C=-\frac{1}{2} \lambda \tau^{2}
$$




$$
\begin{aligned}
& -A \nu^{-(\alpha+1)} \tau^{-(\alpha+1)}(\nu+\tau)+(2 B+C)(\nu+\tau)+2 A- \\
& -2(2 B+C)=-\lambda \nu \tau .
\end{aligned}
$$

We now solve (7.11) for $-A(\nu \tau)^{-(\alpha+1)}$ and substitute the resulting expression into (7.10), obtaining an equation with a factor $v-\tau$. Since we wish to study the locations of possible solutions of (7.1), (7.2) with $\nu \neq \tau$, we cancel this factor obtaining

$$
(B+C)(\nu+\tau)+A-2 B-C=\frac{1}{2} \lambda \tau^{2} \text {. }
$$

Let us denote the solution of (7.12) for $v$ in terms of $\tau$ by $\nu^{\#}(\tau, \lambda)$. If we replace $\nu$ in $(7.10)$ with $\nu^{\#}(\tau)$, we obtain

$$
A \tau^{-(\alpha+1)}=(\lambda \tau-C)\left[\nu^{\#}(\tau, \lambda)\right]^{\alpha+1}
$$

For $\lambda>0$, trivial solutions of our boundary value problem are characterized by $\nu=\tau=k(\lambda) \varepsilon(0,1)$, $N=T=-\frac{1}{2} \lambda k(\lambda)^{2}$. We accordingly limit our attention to a neighborhood (possibly quite large) of the trivial solutions by seeking solutions of (7.13) for which

$$
\begin{aligned}
& (7.14 a, b, c, d) \quad \tau \varepsilon(0,1), \nu^{\#}(\tau, \lambda) \varepsilon(0,1), \\
& \quad \mathrm{N}\left(\nu^{\#}(\tau, \lambda), \tau\right)<0, \quad \mathrm{~T}\left(\nu^{\#}(\tau, \lambda), \tau\right)<0
\end{aligned}
$$

for $\lambda>0$. We wish to characterize where (7.13) has solutions satisfying (7.14) . Such solutions lie in the 
complement of $G$. (If (7.14) is not imposed, then (7.13) always has a solution.)

Note that $(7.14 \mathrm{c})$ is automatically satisfied by virtue of (7.1). We now use (7.12)-(7.14) to show that the set of points $(\nu, \tau) \varepsilon(0,1) \times(0,1)$ at which $(7.1)$, (7.2) has solutions for $(7.8),(7.9)$ can be quite small. Call this set $H(\lambda)$. Clearly

$$
\begin{aligned}
& H(\lambda) \equiv\left\{(\nu, \tau) \varepsilon(0,1) \times(0,1): \nu=\nu^{\#}(\tau, \lambda),\right. \\
& \left.\tau \text { satisfies }(7,13), \quad T\left(\nu^{\#}(\tau, \lambda), \tau\right)<0\right\} .
\end{aligned}
$$

We now obtain a number of inequalities that elements of $H(\lambda)$ must satisfy .

Equation (7.3) implies that $(7.14 d)$ is satisfied

if and only if

$$
v^{\#}(\tau, \lambda)>\tau / 2 .
$$

Equation (7.12) implies that

$$
v^{\#}(\tau, \lambda)>-\tau+1+\frac{B-A}{B+C}
$$

which can represent a very severe or an insignificant restriction depending on the relative size of $A, B, C$. Equation (7.12) and conditions $(7.14 a, b)$ imply that

$$
\tau \leqq[2(C+A)]^{\frac{3}{2}} \lambda^{-\frac{3}{2}} .
$$


A simple graphical analysis of (7.13) based on $(7.14 a, b)$ shows that

$$
\tau \geqq \min \left(\frac{A+C}{\lambda}, 1\right) \quad
$$

Note that if $B-A$ and $\lambda$ are sufficiently large, then the set of $\tau$ 's satisfying $(7.14),(7.17),(7.18)$ is empty. If $\lambda$ is small enough, then (7.19) implies that $\tau \geqq 1$

$$
\text { By substituting (7.16) into (7.12) we obtain }
$$

$(7.20) \tau \notin\left[\tau_{-}, \tau_{+}\right], \lambda \tau_{ \pm} \equiv \frac{3}{2}(B+C) \pm\left[\frac{9}{4}(B+C)^{2}+2 \lambda(A-2 B-C)\right]^{\frac{1}{2}}$

the interval $\left[\tau_{-}, \tau_{+}\right]$being regarded as empty if $\tau_{ \pm}$are not real. If $\mathrm{A}-2 \mathrm{~B}-\mathrm{C}>0$ and if $\lambda$ is large enough, then (7.20) delivers a lower bound for $\tau$ that is larger than that of (7.19).

We seek similar estimates independent of $\lambda$. Let us use $(7.18)$ to get a lower bound for $A \tau^{-(\alpha+1)}$. A sketch shows that a lower bound for $\tau$ is the solution of

$$
A\left[\frac{2(C+A)}{\lambda}\right]^{-(\alpha+1) / 2}=(\lambda \tau-C) \text {. }
$$

Thus 
$(7.22)$

$$
\tau \geqq \frac{C}{\lambda}+\frac{A \lambda^{(\alpha-1) / 2}}{[2(C+A)]^{(\alpha+1) / 2}}
$$

If $\alpha \varepsilon(0,1)$, then (7.22) delivers a lower bound dependent on $\lambda$, but nevertheless better than those of (7.19) and (7.20) when $\lambda$ is large enough. If $\alpha=1$, then (7.22) gives bound that is clearly better than (7.19) and that itself has a further lower bound independent of $\lambda$. If $\alpha>1$, then

$$
2 \tau \geqq \xi x^{-2}+(1-\xi) x^{\alpha-1}
$$

$$
=\frac{(\alpha+1) C^{(\alpha-1) /(\alpha+1)} A^{2 /(\alpha+1)}}{(\alpha-1)(\alpha-1) /(\alpha+1) 2^{-2(\alpha+2) /(\alpha+1)}(C+A)}
$$

with $x \equiv\left[\frac{2 C}{(\alpha-1) A}\right]^{1 /(\alpha+1)}, \quad \xi=\frac{C}{C+A}$, the expressions on the right side of (7.23) belng the minimum of the right side of (7.22) for $\lambda \in(0, \infty)$. An upper bound for the rightmost term of $(7.23)$ can be obtained by the Young inequality. The right side of $(7.23)$ can be made arbitrarily large by taking $\alpha-1$ sufficiently small and positive.

It is not difficult to obtain further estimates, e.g., estimates for the neighborhood discussed in the comments following $(7.7 \mathrm{~b})$. We refrain from doing so.

The basic message of this section is that the set $G$ can be very large and that prècise information about it can 
be gained directly from degenerate constitutive equations. Moreover, we would be able to strengthen the global results of Section 7 even more by obtaining estimates for the location of solutions of our boundary value problem and restricting $G$ to refect such estimates. We do not attempt such estimates here. 


\section{CONCLUSION.}

In an unshearable shell $n$ is constrained to be zero. The shear resultant $H$, not defined constıtutıvely, is the Lagrange multiplier malntaining the constraint of unshearabllity. It is a fundamental unknown of the constrained problem. The same analysis as that used above can treat unshearable shells if $H$ is treated as $\eta \quad 1 s$.

Some of our techniques are reminiscent of those of Antman (1978) for plates. The difference in boundary conditıons forced us to give the coordinate functions $r$ and $z$ a central role in the analysis of coation 5, which they lacked in the plate theory. The issues treated in section 7 never arose for plates. Of course the nodal properties of branches for shells differ markedly from those for plates and for circular arches (cf. Antman \& Dunn, (1980).) Incidentally, the uniqueness theorem of Antman (1978, Section 7) is faulty; it can be readily corrected by followind the development of section 6 above.

It is illuminating to compare our results with the numerical results of Bauer, Reiss, \& Keller (1970). Treating an engineering theory of shells they discovered the remarkable fact that all the bifurcating branches they analyzed are connected. Our results point in the diametrically opposite direction. We showed that nodal properties of branches bifurcating from simple eigenvalues could only lose their nodal structure at certain special places in solution- 
parameter space. In particular, if we could obtain delicate pointwise estimates showing that $\nu \varepsilon(0,1), \tau \varepsilon(0,1)$, $\mathrm{N}<0, \mathrm{~T}<0$, everywhere on a famıly of bifurcating branches, then the results of Sections 6 and 7 show that nodal properties cannot switch along such branches. Consequently such branches could not connect bifurcation points with simple eigenvalues having different eigenfunctions. Now Section 7 shows that for fixed $\lambda$ the complement of $G$ is generically a finite number of points. On the basis of such genericity arguments we could expect that on a given bifurcating branch there would be no $s_{0} \in[0, \pi]$ and $\lambda>0$ such that $\left(\nu\left(s_{0}\right), \tau\left(s_{0}\right), \lambda\right) \notin G$. ( Such genuric1ty arguments are dangerous: The nodal structure we found for $n$ and $\psi-s$ is not generic for pairs of functions. I The chief source of the suspected varlance between our results and those of Bauer, Reiss, \& Keller is likely the fact that various geometric terms regarded as small were discarded in their theory ( as is typical of all tech". nical shell theories). Consequently these terms could not come into play in the large solutions they computed. A concelvable alternative explanation of the connectedness of all the bifurcating branches found by Bauer, Reıss, \& Keller is that they correspond to spurious solution branches of the sort often found in numerical studies of bifurcation problems. We tend to dismiss this explanation because it 
does not seem compatible with the consistent pattern they found. Bauer, Reiss, \& Keller did not qlve enough data for us to determine what sort of nodal properties their solutions possess.

Our approach may also be compared with that of Lange \& Kriegsmann (1981) who studied continuation properties for very thin shells by using asymptotic expansions. Our global bifurcation theory gives an approach to the continuation of branches that does not require special hypotheses on thickness, or more generally, on constitutive functions. Presumably their detalled approach could be applied to our geometrically exact theory for constitutive functions corresponding to very thin shells. We could also introduce the thickness $h$ as a second bifurcation parameter and study the role of thickness by using global multiparameter bifurcation theory (cf. Alexander \& Antman (1981)).

We mention that the perturbation results of Shih (1985) show that the local disposition of bifurcating branches depends critically on the nature of our nonlinear constitutive equations. In particular, the local structure can be quite different from that found in technical shell theories, which essentıally use linear constıtutive relations.

As we have indicated above, the process of numerically constructing solution branches can be quite tricky. It would seem that nodal properties on solution branches could give an extra control on numerical computation. But, to our knowledge, no such use of nodal properties lias been attempted. 
Acknowledgement. Part of the work presented here was developed by Shih (1985) for his doctoral dissertation in the Department of Mathematics of the University of Maryland. The work of Antman was partially supported by a arant from the National Science Foundation. 
§9. APPENDIX A. FORMULATION OF THE GOVERNING EQUATIONS.

In this appendix we describe a special director theory for a nonlinearly elastic axisymmetric shell undergoing an axisymmetric deformation. Let $\{\underset{\sim}{i}, \underset{\sim}{j}, \underset{\sim}{k}\}$ be a right-handed orthonormal basis for the Euclidean space $\mathrm{E}^{3}$.

In the special Cosserat theory we employ, a shell is regarded as a surface together with a unit vector field, called the director field, defined on it. For such an axisymmetric shell undergoing an axisymmetric deformation, the surface is generated by a plane curve rotated about the k-axis and the director field is likewise generated by rotating a director field coplanar with the generating curve.

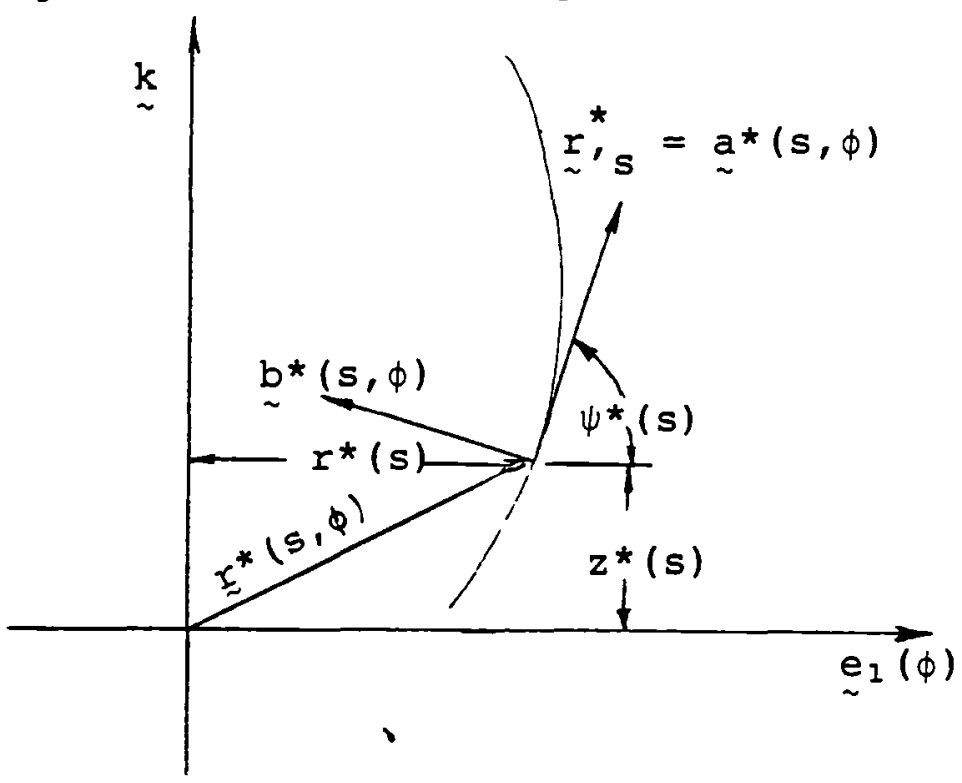

Fig. 9-1

Reference Configuration

Let

$$
\underset{\sim}{e_{1}}(\phi)=\cos \phi \underset{\sim}{i}+\sin \phi \underset{\sim}{j}
$$

$$
{\underset{\sim}{e}}_{2}(\phi)=-\sin \phi \underset{\sim}{i}+\cos \phi \underline{\sim}
$$


The position ${\underset{\sim}{r}}^{*}(s, \phi)$ of a typical material point on the (mid)surface of an axisymmetric shell in its undeformed reference configuration is (see Fig. 9-1),

$$
\underset{\sim}{r}(s, \phi)=r *(s) \underset{\sim}{e_{1}}(\phi)+z *(s) \underset{\sim}{k},
$$

where $\phi$ is the meridional angle and $s$ measures arc length parameter. $(s, \phi)$ identify each material point of the (mid)surface. We let $s \in[0, I]$. Define

$$
\underset{\sim}{a}(s, \phi)=\cos \psi^{*}(s) \underset{\sim}{e_{1}}(\phi)+\sin \psi^{*}(s) \underset{\sim}{k},
$$

$$
\underset{\sim}{b}(s, \phi)=-\sin \psi^{*}(s) \underset{\sim}{e_{1}}(\phi)+\cos \psi^{*}(s) \underset{\sim}{k}
$$

where $\psi^{*}(\mathrm{~s})$ is the angle between the direction of the tangent vector to the curve ${\underset{\sim}{*}}^{*}(\cdot, 0)$ at $s$ and that of ${\underset{\sim}{e}}_{1}(\phi)$.

\section{Deformed Configuration}

The axisymmetrically deformed configuration of an axisymmetric shell is determined by a pair of continuously differentiable functions

$$
[0, L] \times[0,2 \pi] \ni(s, \phi) \rightarrow \underset{\sim}{r}(s, \phi), \underset{\sim}{b}(s, \phi)
$$

where

$$
\underset{\sim}{r}(s, \phi)=r(s) \underset{\sim}{e_{1}}(\phi)+z(s) \underset{\sim}{k},
$$

$$
\underset{\sim}{\mathrm{b}}(s, \phi)=-\sin \psi(s) \underset{\sim}{e_{1}}(\phi)+\cos \psi(s) \underset{\sim}{\mathrm{k}} \text {. }
$$


Define

$$
\underset{\sim}{a}(s, \phi)=\cos \psi(s) \underset{\sim}{e_{1}}(\phi)+\sin \psi(s) \underset{\sim}{k},
$$

$$
\underset{\sim}{r} s(s, \phi)=v(s) \underset{\sim}{a}(s, \phi)+\eta(s) \underset{\sim}{b}(s, \phi),
$$

$$
\underline{\sim}_{1 \phi}(s, \phi)=r(s) \underset{\sim}{e_{2}}(\phi),
$$

where $r_{\sim}(s, \phi)$ and ${\underset{\sim}{r} \phi}_{\phi}(s, \phi)$ are partial derivatives of $r(s, \phi)$ with respect to $s$ and $\phi \cdot$ (See Fig. 9-2)

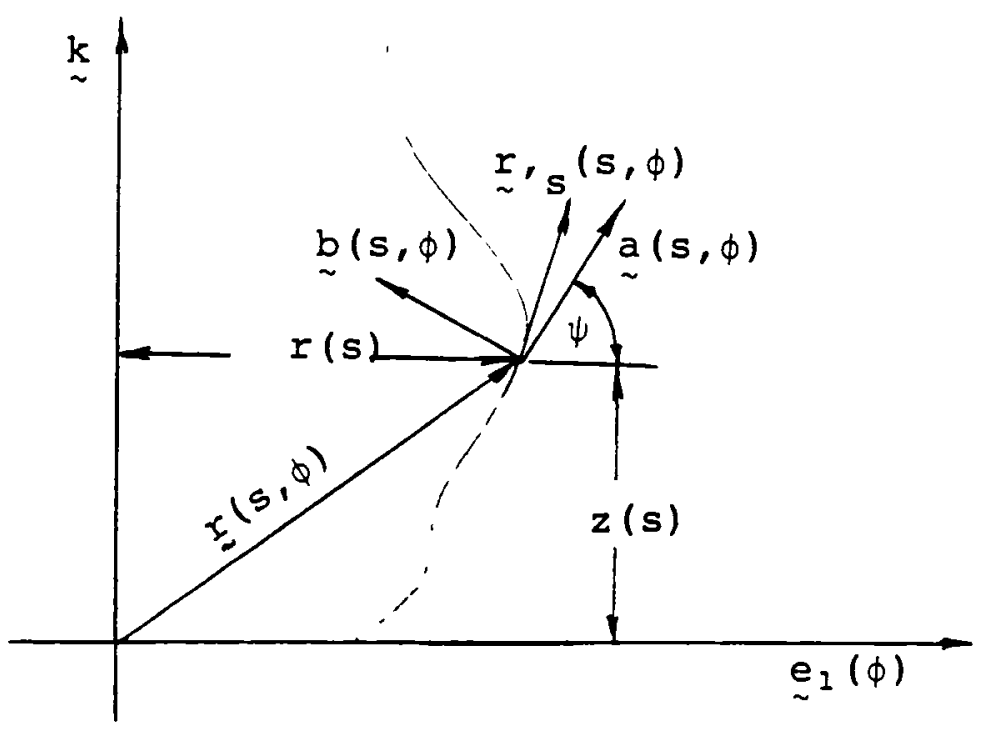

Fig. 9-2.

Deformed Configuration

The strain variables ( which do not all vanish in the reference configuration ) consıst of invariants ( namely the scalar product and scalar triple products) of

$$
\begin{aligned}
& \underset{\sim}{r_{s}}(s, \phi)=\underset{\sim}{\operatorname{va}}(s, \phi)+\underset{\sim}{\eta \underset{b}{b}}(s, \phi) ; \\
& \underset{\sim}{b_{s}}(s, \phi)=-\psi^{\prime} \underset{\sim}{a}(s, \phi) ;
\end{aligned}
$$




$$
\begin{aligned}
& \underline{\sim}_{\phi}(s, \phi) / r^{*}(s)=\left[r(s) / r^{*}(s)\right] \underset{\sim}{e_{2}}(\phi) ; \\
& \underline{\sim}_{b_{\phi}}(s, \phi) / r^{*}(s)=-\left[\sin \psi(s) / r^{*}(s)\right] \underset{\sim}{e_{2}}(\phi) .
\end{aligned}
$$

These invariants depend only on

where

$$
\underline{w}(s)=(\nu, \tau, n, \mu, \sigma)
$$

- (9.13)

$$
\begin{aligned}
& \tau(s)=r(s) / r^{*}(s) ; \\
& \mu(s)=\psi^{\prime}-\left(\psi^{*}\right)^{\prime} ; \\
& \sigma(s)=\sin \psi(s) / r^{*}(s)-\sin \psi^{*}(s) / r^{*}(s)
\end{aligned}
$$

and the prime denotes the derivative with respect to $\mathbf{s}$.

REMARKS. (1) In the case of a spherical shell of radius 1 , where $\psi^{*}(s)=s, r^{*}(s)=\sin s$, we have $\nu=\tau=1$, $n=0, \sigma=\mu=0$ in the reference configuration. Thus $\nu-1$ is a measure of longitudinal strain, $\tau-1$ the meridional strain, $n$ the shear strain and $\sigma$ and $\mu$ are the clrcumferential and longitudinal bending strains respectively. Note that $\mu+1$ and $\sigma+1$ are almost curvatures.

(2) In the uniform contraction and expansion of a spherical shell into one of different radius, $v-1$ and $\tau$ - 1 change but $\mu$ and $\sigma$ remain zero, so that $\mu$ and $\sigma$ isolate the effects of bending. 
EQUIIIBRIUM EQUATIONS.

We suppose the shell is subjected to a uniform external hydrostatic pressure of constant intensity $\lambda$ per unit actual area. Accordingly, the corresponding force per unit reference area of the shell is

$$
\lambda \frac{\stackrel{r}{r}, s^{\times} \underset{\sim}{r}, \phi}{|\underset{\sim}{r}, s||\underset{\sim, \phi}{r}|} \cdot \frac{\underset{\sim}{r}, s \times \underset{\sim}{r}, \phi}{\left|\underline{\sim}^{*}, s^{\times} \underset{\sim}{r}, \phi\right|}=\lambda \frac{\underset{\sim}{r}, s \times \underset{\sim}{r}, \phi}{r^{*}}
$$

Let

$\Omega_{1}$ be contact force resultant per unit reference length acting across $s=$ constant.

$\underline{\mathrm{n}}_{2}$ be contact force resultant per unit reference length acting across $\phi=$ constant.

$\mathrm{m}_{1}$ be contact couple resultant per undt reference length acting across $s=$ constant.

$\mathrm{m}_{2}$ be contact couple resultant per unit reference length acting across $\phi=$ constant.

The equilibrium of total force on the material of $\left\{(s, \phi): s_{1} \leq s \leq s_{2} ; \phi_{1} \leqq \phi \leqq \phi_{2}\right\} \quad$ implies

$(9.18) \int_{\phi_{1}}^{\phi_{2}}\left[\sim_{1}\left(s_{2}, \phi\right) r^{*}\left(s_{2}\right)-\underline{\sim}_{1}\left(s_{1}, \phi\right) r^{*}\left(s_{1}\right)\right] d \phi+$

$$
\begin{aligned}
& +\int_{s_{1}}^{s_{2}}\left[\underset{\sim}{n_{2}}\left(s, \phi_{2}\right)-\underset{\sim}{n_{2}}\left(s, \phi_{1}\right)\right] d s+ \\
& +\int_{\phi_{1}}^{\phi_{2}} \int_{s_{1}}^{s_{2}} \frac{\stackrel{r_{1}}{r_{s} \times \underset{\sim}{r_{1}} \phi}}{r^{*}} r^{*} d \phi d s=\underline{0} .
\end{aligned}
$$


If $\mathrm{n}_{1}$ and $\mathrm{n}_{2}$ are continuously differentiable, we can differentiate $(9.18)$ with respect to $\phi_{2}$ and $s_{2}$ and then replace $\phi_{2}$ and $s_{2}$ with $\phi$ and $s$ to get

$$
\begin{aligned}
& {\left[r^{*}(s) \underset{\sim}{n_{1}}(s, \phi)\right], s+\frac{\partial \tilde{\sim}_{2}}{\partial \phi}(s, \phi)+\lambda \underset{\sim}{r}, s(s, \phi) \times} \\
& \times \underset{\sim}{r} r_{\phi}(s, \phi)=\underset{\sim}{0} .
\end{aligned}
$$

The equilibrium of total moments on the same shell element implies

$(9.20)$

$$
\begin{aligned}
& \left.\int_{\phi_{1}}^{\phi_{2}}\left(\underset{\sim}{r} \times \underset{\sim}{n_{1}}+\underset{\sim}{m_{1}}\right) r^{*}(s)\right|_{s_{1}} ^{s_{2}} d \phi+\int_{s_{1}}^{s_{2}}\left(\underset{\sim}{r} \times \underset{\sim}{n_{2}}+\underset{\sim}{m_{2}}\right) \\
& \left.\left.\right|_{\phi_{1}} ^{\phi_{2}} \mathrm{ds}+\lambda \int_{\phi_{1}}^{\phi_{2}} \int_{s_{1}}^{s_{2}} \underset{\sim}{r} \times \underset{\sim}{r} \underline{s}_{s} \times \underset{\sim}{r_{1}}\right) d s d \phi=\underset{\sim}{0} .
\end{aligned}
$$

Thus, if $\underset{\sim}{m_{1}}$ and $\underset{\sim}{m}$ are continuously differentiable, we obtain

$$
\begin{aligned}
& {\left[\underset{\sim}{r} \times \underset{\sim}{n_{1}} r^{*}(s)\right]_{, s}+\left[r^{*}(s) \underset{\sim}{m_{1}}\right]_{s_{s}}+\left(\underset{\sim}{r} \times \underset{\sim}{n_{2}}+\underset{\sim}{m_{2}}\right)_{\prime_{\phi}}+} \\
& +\lambda \underset{\sim}{r} \times\left(\underset{\sim}{r}, s \times \underset{\sim}{r}{ }^{\prime} \phi\right)=\underset{\sim}{0} .
\end{aligned}
$$

Substituting (9.19) into (9.21), we get

$$
\begin{aligned}
& {\left[r^{*}(s) \underset{\sim}{m_{1}}(s, \phi)\right], s+\underset{\sim}{r} s_{s}(s, \phi) \times r *(s) \underset{\sim}{n_{1}}(s, \phi)+} \\
& +\underset{\sim}{m_{2}{ }^{\prime}}=\underset{\sim}{0} .
\end{aligned}
$$


We seek axisymmetric solutions, for which we require

$(9.23)$

$$
\underset{\sim}{n_{1}}(s, \phi)=\hat{N}(s) \underset{\sim}{a}(s, \phi)+\hat{H}(s) \underset{\sim}{b}(s, \phi)
$$

$$
\underset{\sim}{n_{2}}(s, \phi)=\hat{T}(s) \underset{\sim}{e_{2}}(\phi)
$$

$(9.25)$

$$
\underset{\sim}{m_{1}}(s, \phi)=-\hat{M}(s) \underset{\sim}{\underset{2}{e}}(\phi)
$$

$(9.26)$

$$
\underline{\sim}_{2}(s, \phi) \cdot{\underset{\sim}{e}}_{2}=0
$$

Thus

$$
\underset{\sim}{m_{2}}=\left(\underset{\sim}{m_{2}} \cdot \underset{\sim}{a}\right) \underset{\sim}{a}+\left(\underset{\sim}{m_{2}} \cdot \underset{\sim}{b}\right) \underset{\sim}{b} \text { with components regarded }
$$
independent of $\phi$. Then

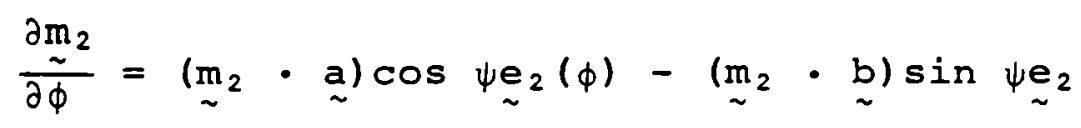

$$
\begin{aligned}
& =\underset{\sim}{k} \times \underset{\sim}{m_{2}} \text {. }
\end{aligned}
$$

Thus (9.19) and (9.22) become

$$
\begin{aligned}
& \left\{r^{*}(s)[\hat{N}(s) \underset{\sim}{a}(s, \phi)+\hat{H}(s) \underset{\sim}{b}(s, \phi)]\right\}, s-\hat{T}(s) \underset{\sim}{e_{2}}(\phi)+ \\
& +\lambda r(s) \underset{\sim}{r}(s, \phi)]_{s} \times \underset{\sim}{e_{2}}(\phi)=\underset{\sim}{0} .
\end{aligned}
$$

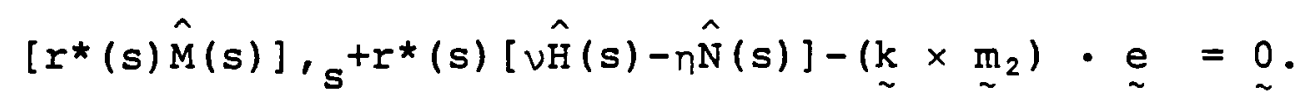

Now we set $\left(\underset{\sim}{m_{2}} \cdot \underset{\sim}{a}\right) \cos \psi(s)-\left({\underset{\sim}{2}}_{2} \cdot \underset{\sim}{b}\right) \sin \psi(s)=\hat{\Sigma}(s) \cos \psi(s)$ 
to obtain

$$
[r *(s) \hat{M}(s)]^{\prime}+r *(s)(\nu \hat{H}-\eta \hat{N})-\hat{\Sigma}(s) \cos \psi(s)=0 .
$$

SPECIALIZATION TO SPHERICAL SHELLS.

Without loss of generality we take the shell radius

to be 1. Thus $r^{*}(s)=\sin s, \psi^{*}(s)=s, z^{*}(s)=-\cos s$, $I=\pi$, and equilibrium equations (9.19a) and (9.22b) become

(9.28a) $[\hat{\mathrm{N}}(s) \sin s]^{\prime}-\hat{\mathrm{T}}(s) \cos \psi(s)-\lambda \operatorname{rn}-\hat{\mathrm{H}}(\mathrm{s}) \sin s \psi^{\prime}(s)=0$ ，

(9.28b) $[\hat{H}(s) \sin s]^{\prime}+\hat{T}(s) \sin \psi(s)+\lambda r \nu+\hat{N}(s) \sin s \psi^{\prime}(s)=0$,

(9.28c) $[\hat{M}(s) \sin s]^{\prime}-\hat{\Sigma}(s) \cos \psi(s)+(\nu \hat{H}-\eta \hat{N}) \sin s=0$.

In order to understand the component $\sum$ occuring in (9.22b), it is convenient to have recourse to the principle of virtual work.

Let $\underset{\sim}{y}(s, \phi), \underset{\sim}{x}(s, \phi) \in C^{2}(\Omega)$ with compact support, where $\Omega=\left[s_{1}, b\right] \times\left[\phi_{1}, B\right], 0 \leqq b \leqq L, 0 \leqq B \leqq 2 \pi$. The principle of virtual work requires that

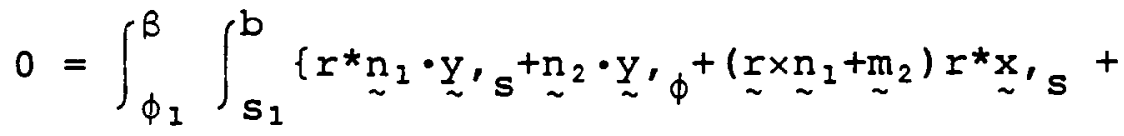

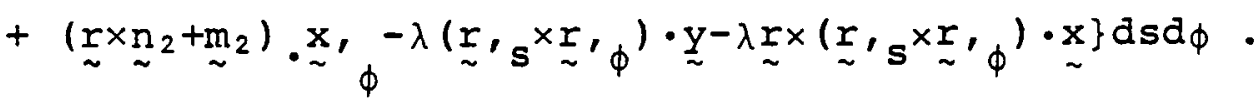


It is convenient to use the traditional notations. Let $\delta \nu, \delta \eta$ and $\delta \psi$ be in $c^{2}(\Omega)$ with compact supports and set

(9.30) $\quad \underset{\sim}{y}=\delta \underset{\sim}{r}-\left(\underset{\sim}{r} \times \sim_{2}\right) \delta \psi ; \underset{\sim}{x}=-\delta \psi \underset{\sim}{e_{2}}$,

where

(9.31)

$$
(\delta \underset{\sim}{r})_{, s}=\delta \underset{\sim}{r}{ }_{s}=\delta \underset{\sim}{v a}+\delta \underset{\sim}{b}+\left(\underset{\sim}{v b}-\sim_{\sim}^{a}\right) \delta \psi
$$

Thus,

(9.32) $\delta \underset{\sim}{r}{ }_{\phi}=\delta{\underset{\sim}{2}}_{2}=(\delta v \cos \psi-\delta \eta \sin \psi-v \sin \psi \delta \psi-n \cos \psi \delta \psi) \underset{\sim}{e_{2}}$.
Also, we get
$(9.33)$

$$
\delta \tau=\frac{\delta r}{r^{\star}}
$$
$(9.34)$
$\delta \sigma=\frac{\delta \psi}{r^{\star}} \cos \psi$,
$(9.35)$$$
\delta \mu=\delta \psi^{\prime},
$$
$(9.36)$

$$
\underset{\sim}{x} \phi \delta{\underset{\sim}{I}}_{1}
$$

Thus (9.29) becomes 
$(9.37)$

$$
\begin{aligned}
0= & \int_{\phi_{1}}^{\beta} \int_{s_{1}}^{b}(N \delta v+H \delta n+T \delta \tau+M \delta \mu+\Sigma \delta \sigma) r^{*} d s d \phi- \\
& -\lambda \int_{\phi_{1}}^{\beta} \int_{s_{1}}^{b}\left(\underset{\sim}{r^{\prime}} s \times \underset{\sim}{r_{\prime} \phi^{\prime}}\right) \cdot \underset{\sim}{r} d s d \phi
\end{aligned}
$$

This is the traditional form of the principle of virtual work with $\Sigma$ as the generalized force corresponding to the strain variable $\sigma$.

Note that in the expression $\left(\underset{\sim}{m_{2}} \cdot \underset{\sim}{a}\right) \cos \psi-\left(\underset{\sim}{m_{2}} \cdot \underset{\sim}{b}\right)$ $\sin \psi=\hat{\Sigma} \cos \psi$, we do not imply that $\hat{\Sigma}=\underset{\sim}{m_{2}} \cdot \underset{\sim}{a}$ and that $\underset{\sim}{b} \cdot \underset{\sim}{m_{2}}=0$. 
9. REFERENCES

J.C. Alexander and S.S. Antman (1981): Global Behavior of Bifurcating Multidimensional Continua of Solutions for Multıparameter Nonlinear Elgenvalue Problems. Arch. Rational Mech. Anal. 76, 339-354.

S.S. Antman (1971): Existence and Nonuniqueness of Axisymmetric Equilibrium States of Nonlinearly Elastic Shells. Arch. Rational Mech. Anal. 40, 329-372.

S.S. Antman \& E.R. Carbone (1977): Shear and Necking Instabilities in Nonlinear Elasticlty, J. Elastlclty, 7 , 125151 .

S.S. Antman \& S.E. Dunn (1980): nualitative Behavior of Buckled Nonlinearly Elastıc Arches, T. Elasticity, 10, $2<5-239$.

S.S. Antman \& G. Rosenfeld (1978): Global Behavior of Buckled States of Nonlinearly Elastic Rods. SIAM. Rev. $\underline{20}$, 513-566.

L. Bauer, E.L. Reiss \& H.B. Keller (1970): Axısymmetric Buckling of Hollow Spheres and Hemi-Spheres. Comm. Pure \& Appl. Math. 23, 529-568.

E. \& F. Cosserat (1909): Théorıe des Corps Déformables. Hermann.

M.G. Crandall \& P.H. Rabinowitz (1971): Bifurcation from Simple Lidenvalues. J. Funct. Anal. 8 , 321-340.

P.M. Fitzpatrick \& J. Pejsachowicz (1985): An Extension of the Leray-Schauder Deqree for fully Nonlinear Elliptıc Problems, MD84-59PF/JP, TR 84-48, University of Maryland, November 1984.

P. Hartman (1964): Ordinary Differcntıal Equaiıons. Wiley, New York.

C.G. Lange \& G.A. Krlegsmann (1981): The Axisymmetric BranchInq Behavior of Complete Spherical Shells. Quart Appl. M.th. $39,145-178$. 
A. Libai \& J.G. Simmonds (1983): Nonlinear Elastic Shell Theory, Advances in Applied Mechnoics, 23, Acalemic Press. 271371 .

P.M. Naghdi (1972): The Theory of Plates and Shells. In Handbuch der Physık VIa/2 (Ed. C. Truesdell) SpringerVerlag, 425-640.

P.H. Rabinowitz (1971): Some Global Results for Nonlinear Elgenvalue Problems. J. Funct. Analy. 7, 487-513.

E. Relssner (1963): On the Equations for Finite Symmetric Leflection of Thin Shells of Revolution. In "Progress in Applied Mechanics" (The Prager Anniversary Volume) D.C. Drucker, ed., Macmiliian, 171-178.

K.-G. Shih (1985), rilobal Bifurcation Problems of Axısymmetrıc Buckling of Complete Spherical Shells, Doctoral Dissertation, University of Maryland. 
End of Document 